\title{
A Review of Oxytocin and Arginine-Vasopressin Receptors and Their Modulation of Autism Spectrum Disorder
}

OPEN ACCESS

Edited by:

Chiara Verpelli,

Istituto di Neuroscienze (CNR), Italy

Reviewed by:

Adife Gulhan Ercan-Sencicek Yale University, United States Andreas Martin Grabrucker, University of Limerick, Ireland

*Correspondence: Gianluca Esposito gianluca.esposito@ntu.edu.sg

Received: 21 September 2017 Accepted: 18 January 2018

Published: 13 February 2018

Citation:

Cataldo I, Azhari A and Esposito G

(2018) A Review of Oxytocin and Arginine-Vasopressin Receptors and Their Modulation of Autism Spectrum Disorder. Front. Mol. Neurosci. 11:27. doi: 10.3389/fnmol.2018.00027

\author{
Ilaria Cataldo 1,2, Atiqah Azhari ${ }^{3}$ and Gianluca Esposito 1,3* \\ ${ }^{1}$ Department of Psychology and Cognitive Science, University of Trento, Rovereto, Italy, ${ }^{2}$ Mobile and Social Computing Lab, \\ Fondazione Bruno Kessler, Trento, Italy, ${ }^{3}$ Division of Psychology, School of Social Sciences, Nanyang Technological \\ University, Singapore, Singapore
}

Oxytocin (OXT) and arginine-vasopressin (AVP) play a key regulatory part in social and affiliative behaviors; two aspects highly compromised in Autism Spectrum Disorder (ASD). Furthermore, variants in the adjacent oxytocin-vasopressin gene regions have been found to be associated with ASD diagnosis and endophenotypes. This review focuses mainly on common OXTr single nucleotide polymorphisms (SNPS), AVPR1a microsatellites and AVPR1b polymorphisms in relation to the development of autism. Although these genes did not surface in genome-wide association studies, evidence supports the hypothesis that these receptors and their polymorphisms are widely involved in the regulation of social behavior, and in modulating neural and physiological pathways contributing to the etiology of ASD. With a specific focus on variants considered to be among the most prevalent in the development of ASD, these issues will be discussed in-depth and suggestions to approach inconsistencies in the present literature will be provided. Translational implications and future directions are deliberated from a short-term and a forward-looking perspective. While the scientific community has made significant progress in enhancing our understanding of ASD, more research is required for the ontology of this disorder to be fully elucidated. By supplementing information related to genetics, highlighting the differences across male and female sexes, this review provides a wider view of the current state of knowledge of OXTr and $A V P r$ mechanisms of functioning, eventually addressing future research in the identification of further risk factors, to build new strategies for early interventions.

Keywords: OXTr, AVPr, rs53576, rs2254298, rs2268493, Autism Spectrum Disorder (ASD)

\section{INTRODUCTION}

Afflicting individuals across ethnic and socioeconomic divides, autism spectrum disorder (ASD) is a severe neurodevelopmental condition known to first emerge in individuals before the age of three (Fombonne, 2009; American Psychiatric Association, 2013). Occurring 4 times more in boys than girls (Baron-Cohen et al., 2005; Steyaert and De la Marche, 2008; Lintas and Persico, 2009), this severe developmental disorder is characterized by three diagnostic symptom domains: impairments in social interaction, verbal and non-verbal communication, and restricted, repetitive patterns of behavior, interests or activities (American Psychiatric Association, 2013). The autistic 
phenotype leaves individuals less adept at normal social interaction (Kanner, 1968; Clifford et al., 2007), expressive language, non-verbal communication (e.g., maintaining eye contact) (Lord et al., 2000; Hill and Frith, 2003; Groen et al., 2008), peer relationships (Kanner, 1968; Clifford et al., 2007) and flexibility in behavioral patterns (Szatmari et al., 2006). Beyond these two broad categories, a diverse constellation of symptoms frequently co-occur with ASD diagnosis. These include hyperactivity (Volkmar et al., 1999; Simonoff et al., 2008), sensory dysfunction (Baranek et al., 2007; Tommerdahl et al., 2007; Cascio et al., 2008; Coskun et al., 2009; Boyd et al., 2010; Kwakye et al., 2011) and intellectual disability (Chakrabarti and Fombonne, 2005). With an estimated heritability of around $64-91 \%$ in twin studies (Tick et al., 2016) and 31$71 \%$ in whole-genome genotyping studies (Gaugler et al., 2014), compelling evidence suggests that the etiology of ASD bears a strong genetic influence (Szatmari et al., 2006; Abrahams and Geschwind, 2008). An array of approaches, including genome-wide association studies, genetic linkage and expression analysis have been utilized to identify individual genes contributing to ASD (Veenstra-VanderWeele and Cook, 2004; Vorstman et al., 2006; Freitag, 2007; Gupta and State, 2007; Abrahams and Geschwind, 2008; Wang et al., 2009). Kumar and Christian (2009) have also reported that numerous types of genetic disorders, from mutations in single nucleotide bases to irregularities in chromosomal structures and numbers, can lead to the development of an autistic phenotype (Buxbaum, 2009).

In addition to complex genetic abnormalities, the quest to uncovering the causes of ASD has been rendered more difficult by the contributing role of environmental factors, including toxins, pesticides in utero exposure to drugs and maternal infection (Moore et al., 2000; Rasalam et al., 2005; St-Hilaire et al., 2012; Volk et al., 2013; Berko et al., 2014; Sandin et al., 2014; Tordjman et al., 2014). Some of these factors might exert their influence during pregnancy (Depino, 2017; Nicolini and Fahnestock, 2017) while others might occur during critical stages of development. One environmental variable that has been wellestablished to increase the risk of ASD is maternal infection during early fetal neurodevelopment (Atladóttir et al., 2010; Zerbo et al., 2013, 2015; Lee et al., 2015; Mazina et al., 2015). Given the wide-ranging detrimental effects of prenatal infection (e.g., rubella and cytomegalovirus) on the central nervous system (Johnson, 1994), it is to no surprise that prenatal infection has emerged as a notable environmental risk factor for ASD. While convergent findings on the role of prenatal infection in ASD development are readily available in the existing literature, numerous differences still prevail in pin-pointing the specific causative agent and exact prenatal period involved. For instance, Atladóttir et al. (2010) found that viral infection in the first trimester, and any infection in the second trimester led to increased risk of ASD. Contrary to the agent- and trimesterspecific associations highlighted in 2010, Lee et al. (2015) later discovered that infectious agents of any type (i.e., bacterial, viral, other infection), during the entire course of pregnancy, can contribute to ASD development. To elucidate the mechanism of action of these infections, numerous animal model studies have been conducted, revealing that prenatal infection, either through direct exposure of preganant mice to viruses, or via activation of maternal immune response in the absence of an infectious agent, led to atypical behaviors in the resulting pups (Brown and Derkits, 2010; Meyer et al., 2011; Patterson, 2011; Miller V. M. et al., 2013). This suggests that infectious agents, albeit possessing individual characteristics, share common biological pathways that influence the development of ASD. One theory that has been gaining recognition in recent years is the cytokine hypothesis. Cytokines, which are signaling molecules in the immune system, play a critical role in neuronal migration, survival and development (Deverman and Patterson, 2009). Maternal infection is hypothesized to trigger a dysregulation in cytokine levels, which disrupts typical neurodevelopment and contributes to ASD (Ashwood et al., 2006, 2011). Beyond the fetal period, environmental factors have also been found to induce epigenetic changes. For instance, intense social experiences in sensitive life stages can have enduring influences not only on behavior, but also on AVPR1a gene expression in the hippocampal region through a change in patterns of methylation (Bodden et al., 2017).

Recent epidemiological studies demonstrate a disturbing upward trend in number of ASD cases, with an estimate of 1 in every 68 children being diagnosed with ASD (NIMH statistics prevalence of autism). Some researchers have proposed this increase to be due to an enhanced awareness of the disorder or a reduced strictness in diagnoses (for example, see Rutter, 2005), while others point to the possibility of a true increase in number of individuals with ASD (Fombonne, 2002; Inglese and Elder, 2009). In light of these prevailing statistics, efforts in the search for the roots of ASD has greatly increased. However, such efforts have generated inconsistent results due to heterogeneity of ASD and the polygenic manner in which various genetic loci contribute to the disorder (Betancur, 2011; Geschwind, 2011). One possible way to parse the heterogeneity of ASD may be through the use of biological biomarkers (Hazlett et al., 2005; Redcay and Courchesne, 2005; Giulivi et al., 2010; Schumann et al., 2010; Courchesne et al., 2011a,b; Rossignol and Frye, 2012). In this review, our focus will be directed at the genetic level, systematically examining the role of single nucleotide polymorphisms (SNPs) in oxytocin receptor $(\mathrm{OXTr})$ and arginine-vasopressin receptor $(A V P r)$ genes in the etiology of ASD. Besides having been established to play an imperative role in social behavior and affiliation (Insel and Shapiro, 1992; Panksepp, 1992; Veenema and Neumann, 2008), OXTr and AVPr have also been previously associated with ASD (Israel et al., 2008). Aside from their involvement in regulating behaviors, this review focuses on these two receptor classes as they exhibit different protein expression patterns in males and females, an essential biological characteristic that ought to be taken into account while approaching strongly sex-biased disorders such as ASD.

\section{Genome-Wide Association Studies vs. Candidate-Gene Studies}

The latest technological advancements in the genetic field has allowed researchers the opportunity to detect specific gene loci involved in neuropsychiatric and developmental disorders. In 
the 1980's, researchers began to consider the possibility of a strong genetic component underpinning ASD. Since then, many genes were found to be associated to autistic traits. Genomewide association studies (GWAS) highlighted the association between autism and genes, from rare ones, such as PARK2 (Yin et al., 2016), to those which are more common, like SHANK3 (Nemirovsky et al., 2015; Sanders et al., 2015; Guo et al., 2017), FOXP1, FHIT, (The Autism Spectrum Disorders Working Group of The Psychiatric Genomics Consortium, 2017) and independent polymorphisms, like rs57709857-A (The Autism Spectrum Disorders Working Group of The Psychiatric Genomics Consortium, 2017). Although this technique efficiently reveals genes involved in autism within a shorter period of time, findings should be interpreted with caution given the great overlap present between genes involved in ASD and other medical/neuropsychiatric conditions. The current literature presents evidence of shared genetic, biological, and physiological etiology between mental disorders, such as ASD and ADHD (Craig et al., 2015; Panagiotidi et al., 2017), schizophrenia (Ishizuka et al., 2017; Prata et al., 2017), major depressive disorder (Glessner et al., 2017), and medical conditions like epilepsy (for a review see Strasser et al., 2017) and disorders of the immune system (Cieślinska et al., 2017; Li et al., 2017). Aside from these considerations, other limiting aspects of GWAS studies include the need for greater sample sizes and difficulties in replicating studies involving a population with ASD. These complexities may be due to the heterogeneous nature of the disorder, differences among ethnicities, and other environmental factors which render genome-wide studies useful but not resolutive.

Candidate gene studies is an alternative approach that requires large sample sizes to explore haplotypes, single genes, or even a few polymorphisms at a time. Some optimistic developments in research of $O X T r$ and $A V P r$ receptor classes have been required through the use of candidate gene studies. For instance, this method has elucidated specific associations of $O X T r$ and $A V P r$ polymorphisms to specific structures of the brain (e.g., amygdala) (Schneider-Hassloff et al., 2016), as well as to downstream physiological pathways such as the hypothalamic-pituitary axis (Auer et al., 2015). Furthermore, this methodology is more applicable to the new criteria of ASD diagnosis found in the latest edition of the Diagnostic and Statistical Manual of Mental Disorders (DSM-5), which regards disorders to exist on a continuous spectrum, ranging from the general population to the severely impaired clinical group.

\section{ASD in the DSM-5}

Autism has been shown to be a heterogeneous and complex condition with multiple etiologies, resulting in a diverse array of clinical presentations across individuals. With revisions in the clustering of symptoms and criteria for diagnosis, DSM-5 has prescribed to the notion of "spectrum," giving importance not only to singular features but also to the extent to which these symptoms impair daily functioning. Ever since this latest DSM edition has been applied to clinical practices, a decrease in number of ASD diagnoses has been observed (Kulage et al., 2014; Sturmey and Dalfern, 2014; Smith et al., 2015). In distinguishing the degree of severity of ASD symptoms, this paradigm shift has established a continuum of autistic traits that ranges from the mild to the severely impaired. DSM-5 more accurately reflects how social behaviors widely differ from person to person, making it possible for autistic traits to be found in the general population too, especially in the case where individuals share common environmental factors (Skylark and Baron-Cohen, 2017; Suzuki et al., 2017). Recently, it has been observed that ASD and autistic traits also share similar genetic and biological elements (Bralten et al., 2017), but it is necessary to take a look at these information emerging from literature holistically, before speculating the mechanisms underlying the development of ASD. The pursuit of research that investigates common autistic characteristics across both clinical and non-clinical populations might be helpful in developing more streamlined diagnostic practices.

\section{Lessons from Animal Models}

In the numerous attempts to define the etiology of autism, much progress has been made possible due to research on animal models, which has exponentially increased since the use of the transgenic mouse. To date, this technique has allowed researchers to manipulate genetic information (Halladay et al., 2009), allowing control over genetic factors that contribute to the development of neurodevelopmental disorders, potentially giving rise to new medical treatments (Avraham et al., 2017). Rodent models have been adopted in ASD studies to investigate mechanisms for prevention (Vuillermot et al., 2017), and potential therapeutic treatments (Chadman, 2017; Guoynes et al., 2017), as well as to better understand the involvement of the gutbrain axis (for a review see Nithianantharajah et al., 2017) and the immune system (Schwartzer et al., 2017) in ASD etiology. To illustrate this, we draw specific attention to the pivotal role that animal models have played in elucidating how maternal immune activation, a well-ascertained environmental risk factor, contributes to ASD. Using immune activating agents, such as polyinosinic-polycytidylic acid (PolyIC), these studies induce an activation of the maternal immune system in pregnant rodents (Medzhitov, 2001). Following that, parameters of ASD-like behaviors in the offspring, such as pup ultrasonic vocalizations (Malkova et al., 2012; Hsiao et al., 2013; Schwartzer et al., 2013; Choi et al., 2016) and brain abnormalities, are compared against parallel ASD characteristics in humans (for reviews, see Meyer et al., 2009; Boksa, 2010). Cross-species evaluation has generated interest in examining the evolutionarily conserved effects of maternal immune activation on ASD-like behaviors (Belzung and Lemoine, 2011; Stewart and Kalueff, 2015) in rat and nonhuman primate models as well. While mice models have been the long-standing preferred species in biomedical research due to the extensive range of possible genetic manipulations, rat models feature a more complex array of brain activities and enriched social behaviors (Yee et al., 2012; Vanderschuren and Trezza, 2013; Ku et al., 2016), that serve as an advantage for studies relating to autism, since this disorder primarily features a deficiency in social cognition (Couture et al., 2010). Despite the numerous advantages that the rodent species has to offer, the social signals (i.e., vocalizations, facial expressions, body gestures) and underlying patterns of brain activity in rodents are limited (Chang et al., 2013). Thus, non-human primate models, 
such as the rhesus monkey, bridges the link from rodent models to humans (Watson and Platt, 2012; Platt et al., 2016), although such studies may be rife with ethical concerns.

With respect to OXT and AVP systems, evidence in literature highlights the contribution of these two peptides in social processes (Modi and Young, 2012; Peñagarikano et al., 2015; Zimmermann et al., 2016). Engineering of animal models specific to genes implicated in these systems is crucial in aiding the investigation of ASD etiology from this research front (Hammock and Young, 2006; Murakami et al., 2011; Peñagarikano, 2017). Indeed, OXT and OXTr knockout (KO) mice have been found to exhibit abnormal social behaviors akin to ASD-like traits (Ferguson et al., 2000; Winslow and Insel, 2002) and futher studies have begun to uncover the specific biological pathways involved. For example, Fujiwara et al. (2016) have recently investigated the role of HPC-1/syntax-in1A (STX1A), a $\mathrm{N}$-ethylmaleimide-sensitive fusion attachment protein receptor complex which regulates the synaptic transmission of OXT (Südhof and Rothman, 2009). Employing a social novelty preference test, they found that STX1A KO mice showed unusual social behavior that is thought to be a component of a stereotypic ASD-like behavioral profile (Burket et al., 2011), also displayed by OXTR KO mice (Fujiwara et al., 2010). However, intracerebroventricular administration of oxytocin (OXT) partially rescued this phenotype (Fujiwara et al., 2016). This is a prime depiction of how animal model studies illuminate biological pathways related to the ASD phenotype as well as gives insight into preclinical research options that potentially advances the treatment of this disorder.

\section{OXTr and AVPR}

The core symptoms of autism revolve around deficits in normal social communication (Snow et al., 2009; Sala et al., 2011). Two neuropeptides of interest, oxytocin (OXT) and argininevasopressin $(A V P)$, have been well-documented to mediate social and cognitive processes in clinical and non-clinical settings (Neumann, 2008; Ebstein et al., 2009, 2012), as well as across species (Veenema and Neumann, 2008; Ebstein et al., 2009; Zhang et al., 2017). They have been established to facilitate biologically adaptive social behaviors, such as trust (Kosfeld et al., 2005) positive communication (Ditzen et al., 2009), maternal care (Bielsky et al., 2005; Bosch and Neumann, 2008), and affiliation (Lim et al., 2005; Veenema et al., 2010). Because of their integral function in shaping social conduct, researchers began to postulate the involvement of these neuropeptides in the etiology of ASD (Insel et al., 1999; Hammock and Young, 2006; Watanabe et al., 2017). Indeed, animal model studies have shown that administration of OXT and AVP were able to rescue autistic traits (Sala et al., 2011) and increase social recognition memory (Bielsky and Young, 2004; Winslow and Insel, 2004), while in humans, intranasal OXT has been reported to heighten the understanding of others' mental states (Domes et al., 2007) a deficit found amongst individuals with ASD (Caronna et al., 2008). As these neuropeptides exert their behavioral effects upon binding to three specific G-protein-coupled (Gimpl and Fahrenholz, 2001; Thibonnier et al., 2002) receptors; OXTr, AVPR1a, and AVPr1b (Manning et al., 2008), the next advance in this field was made when scientists looked toward $O X \operatorname{Tr}(\mathrm{Wu}$ et al., 2005; Jacob et al., 2007; Lerer et al., 2008) and $A V \operatorname{Pr}$ (Kim et al., 2002; Wassink et al., 2004; Yirmiya et al., 2006) as potential candidate genes (for a review, see Zhang et al., 2017).

Known for its role in facilitating social behavior, OXTr has been implicated in neuropsychiatric disorders, ranging from social anxiety disorders and schizophrenia (Wu et al., 2005; Jacob et al., 2007; Lerer et al., 2008; Yrigollen et al., 2008; Ebstein et al., 2009; Gregory et al., 2009; Liu et al., 2010; Guastella and MacLeod, 2012; Meyer-Lindenberg and Tost, 2012) to autism (Wu et al., 2005; Ylisaukko-oja et al., 2006; Jacob et al., 2007; Lerer et al., 2008; Yrigollen et al., 2008; Gregory et al., 2009; Liu et al., 2010; Campbell et al., 2011). Based on numerous genome-wide association studies, $O X T r$ has been proposed to be a potential risk gene in ASD. Supporting this proposition is a $0.7 \mathrm{Mb}$ deletion in 3p25.3, which has been found to occur with autism (see Figure 1). Since OXTr was encompassed in the deleted region, such mutations implicating OXTr were suggested to be involved in ASD etiology (Wu et al., 2005; Jacob et al., 2007; Lerer et al., 2008). However, this phenomenon appears to be rare as other studies have not reported OXTr deletions typifying autism (Sebat et al., 2007; Marshall et al., 2008; Glessner et al., 2009; Sanders et al., 2015). As compared to deletions, the association of $\mathrm{OXTr}$ SNPs with ASD has been more commonly observed. Several SNPs of OXTr have been linked to ASD, including rs237887, rs2268491 and rs225429, rs7632287 (Ylisaukko-oja et al., 2006; Jacob et al., 2007; Lerer et al., 2008; Yrigollen et al., 2008; Ebstein et al., 2009; Liu et al., 2010; Campbell et al., 2011; Walum et al., 2012; LoParo and Waldman, 2015). However, some studies did not relate any association (Tansey et al., 2010; Wermter et al., 2010; Verhallen et al., 2017), while others reported ethnic differences in the association of OXTr SNPs with autism (Wu et al., 2005; Jacob et al., 2007; Lerer et al., 2008; Liu et al., 2010).

In the domain of social behavior, AVPR1a (Lim et al., 2005; Veenema et al., 2010) and AVPR1b are two integral players from the AVP system. Well-known to be located on chromosome 12q14-15, the gene for AVPR1a possesses three microsatellites in the $5 \mathrm{u}$ flanking region and a fourth one in an intronic region (Thibonnier et al., 2000; Thibonnier, 2004) (see Figure 2). Expression patterns of AVPR1a in the brain significantly predict differences in social behavior of voles within and across species (Hammock and Young, 2002, 2006). Of clinical relevance, AVPR1a polymorphism has been shown to be involved in social recognition (Bielsky et al., 2005), amygdala activation (MeyerLindenberg et al., 2009) and implicated in ASD (Kim et al., 2002; Wassink et al., 2004; Yirmiya et al., 2006). In 2002, Kim et al. (2002) was the first group to document the significant transmission disequilibrium of a microsatellite on AVPR1a and autism, before Wassink et al. (2004) and Yirmiya et al. (2006) independently reproduced this finding. (Israel et al., 2008) found that deficits in socialization skills mediated this link. For the past decade, the central wellspring of research in AVPr lies in the promoter region of two microsatellites, RS1 and RS3, where length of microsatellites has been found to modulate social behavior in human and lower mammals (Israel et al., 2008; Knafo et al., 2008). This observation was supported by corresponding mRNA analyses, in which longer AVPR1a RS3 repeats were found 
to be associated with higher levels of AVPR1a mRNA expression (Knafo et al., 2008). This asserts the functional role of different AVPR1a microsatellites in determining social behavior. Of less prominence in the study of autism is the receptor $A V P R 1 b$, which has been previously linked to aggression, anxiety, pro-sociality and autistic traits (Dempster, 2007; Chakrabarti et al., 2009; Dempster et al., 2009; Zai et al., 2012; Wu et al., 2015). Only recently, Francis et al. (2016) reported a significant association between $A V P R 1 b$ and ASD.

Having chronicled the consensus and contention surrounding OXTr and AVPr in ASD, we have established the foundation for this review. Thereafter, we will endeavor to highlight specific polymorphisms of $O X T r$ and $A V P r$ that have been either recently or repeatedly associated with autism. Exploring the breadth of the current literature, we will provide suggestions on how inconsistencies can be approached and reconciled, as well as recommend possible future directions that might advance this field of research further.

\section{METHODS}

Pubmed, PsycINFO and Scopus databases were used to browse for articles on the oxytocin receptor and arginine vasopressin systems. We comparatively analyzed the entire literature up to December 2017, combining different keywords and Boolean operators (see Figure 3). Firstly, we collated all papers that were generated by the term ["OXTr" OR “AVPr" AND “ASD”], before adding the results of a new search ["oxytocin receptor" OR “arginine vasopressin receptor" AND “autism”]. 176 papers were generated through Pubmed, and 495 and 350 papers were obtained from PsycINFO and Scopus respectively. After duplicates were removed, results from this search amounted to a list of 534 records. Twenty-eight records were removed as they were not full-text citations (i.e., comments, letters), leaving 506 articles to be assessed for eligibility. After filtering for relevant papers based on whether they contained information on "AVPr," "OXTr," and "ASD," 87 studies remained and were included in the qualitative analysis (see Table 1 for the list of papers included in the review). These papers were sub-categorized into different lists according to fixed criteria: receptor in the text (oxytocin and/or arginine vasopressin) related to the ASD topic, species discussed (human/animal), and SNP involved in the research. With regard to $A V P r$, we further subdivided the papers according to both the specific receptor group and the main polymorphism that has been found to be related to the risk of developing ASD; these are AVPR1a (microsatellites RS1 and RS3 mainly) and AVPR1b (polymorphisms rs28632197 and rs3536969). As for $O X T r$, we divided the papers based on the SNPs which were investigated (rs53576, rs2254298, rs2268493). The following section will explore, in greater detail, how SNPs of these receptors contribute to the development of autism, with a specific aim of illustrating how this dual modulation underlies autistic traits in clinical and non-clinical populations.

Oxytocin exerts its impact not only on the central nervous system, where it modulates functions that are relevant to the regulation of social behaviors, but also on autonomic responses, playing a role in motor, metabolic, sensory and visceral systems (Herman, 2012). It is largely expressed in the paraventricular nucleus (PVN) of the hypothalamus, an area where information related to cardiovascular and stress regulation converge and are relayed to downstream pathways, with subsequent effects on activities of the autonomic nervous system and hypothalamicpituitary-adrenal (HPA) axis. It has been shown that from the PVN, oxytocin molecules maneuvre a preferential path to the amygdala, where it modulates emotional functions (Stoop, 2012).

\section{OXTr SNPs and Autism}

There is robust evidence to posit that oxytocin receptor gene plays a determining role in social behaviors, ranging from individual differences in non-clinical populations to ASD. Many studies have been conducted in the past two decades to better understand which polymorphisms have a bigger impact in the development of autism. A pioneer study by Horvath and colleagues found a significant correlation between A carriers in OXTr SNPs rs53576 and rs2254298 and autism (Horvath et al., 2001). Subsequently, other authors expanded our understanding of this relationship (LoParo and Waldman, 2015), finding a correlation between oxytocin receptor gene variants (rs53576, rs2254298 mainly) and autistic traits (Wu et al., 2005; Jacob et al., 2007; Lerer et al., 2008).

For OXTr rs53576, it has been demonstrated that A carriers, who are considered more vulnerable compared to $G$ carriers, display reduced hypothalamic volume, that is shown to be related to susceptibility in ASD development (Liu et al., 2010; Tost et al., 2010; Jack et al., 2012; Wang et al., 2013). Many researchers have contributed studies which revealed the influence of this polymorphism on different social behavior. Specifically, there are evidences of correlations between rs53576 and prosocial disposition (Tost et al., 2010; Kogan et al., 2011; Yamasue, 2013), emotional withdrawal in psychotic disorders (Haram et al., 2015), negative affect (Lucht et al., 2009; Saphire-Bernstein et al., 2011), and reaction to stress and empathy (Rodrigues et al., 2009; Chen et al., 2011; Weisman et al., 2015; McDonald et al., 2016). Indeed, rs53576 has received much attention by researchers in the field of autism, with results suggesting that its involvement is not limited to ASD alone, but influences a wide spectrum of phenotypes related to general sociability and affiliative behaviors (LoParo and Waldman, 2015; Zhang et al., 2017). Generally, these results suggest that rs53576 is more related to sensitivity to social environment than to ASD itself (Tost et al., 2010; Jack et al., 2012; Wang et al., 2013).

Presently, studies on rs53576 have begun to investigate the role of epigenetic modification. For instance, Rijlaarsdam and colleagues have has recently discovered a significant interaction between $O X T r$ methylation and child autistic traits, as well as social communication problems (Rijlaarsdam et al., 2017). Specifically, greater $O X \operatorname{Tr}$ methylation was found to be correlated with social communication problems in rs53576 G-allele homozygotes, rather than A-allele carriers. Such findings encourage us to question the link that has been previously established between ASD susceptibility and the A-allele. This underscores the pertinence of accounting for epigenetic data in understanding the role of $O X \operatorname{Tr}$ polymorphisms in ASD. 
Another well-investigated polymorphism of $O X T r$ is rs2254298, which is located in the third intron, with a guanine to adenine variation that is supposedly the "risk-inducing" form. As with rs53576, several studies attempted to understand the impact of rs2254298 on the development of ASD. Neuroimaging studies revealed a correlation between this variation and increased amygdala volume in general (Furman et al., 2011; Brüne, 2012) and when combined with environmental factors of early life stress (Marusak et al., 2015). Starting from the first genetic study on the etiology of autism in a Chinese population ( $\mathrm{Wu}$ et al., 2005), others followed suit to find a significant correlation between rs2254298 and ASD diagnosis in Caucasian and Japanese samples (Jacob et al., 2007; Liu et al., 2010; LoParo and Waldman, 2015). Other findings centered on OXTr rs2254298 show that autistic traits are related to this polymorphism, but only when it is comprised in a haplotype (Lerer et al., 2008; Mosconi et al., 2009; Nyffeler et al., 2014; Francis et al., 2016). Besides autism, rs2254298 was also linked to social impairment in general (Parker et al., 2014), empathy (Wu et al., 2012) anxiety and depression (Mosconi et al., 2009; Thompson et al., 2011; Bittencourt Jacondino et al., 2014).

On the contrary, some authors found no significant correlation between OXTr rs2254298 and etiology of autism (Tansey et al., 2010; Campbell et al., 2011; Montag et al., 2017). An enlightening meta-analysis by Bakermans-Kranenburg and van Ijzendoorn emphasized the need to account for epigenetic mechanisms when examining SNPs like rs2254298 due to the lack of available research in the literature relating epigenetics not only to ASD development but to general social behavior (BakermansKranenburg and van Ijzendoorn, 2014). Indeed, present data on genetic polymorphisms, up to the year 2014, fail to explain a considerable part of social behavior in humans. Another recent large study found no association between common variants of OXTr and $A V P r$, and social integration (Chang et al., 2014), although the authors suggested that the results could have been small. Additionally, the study was run on healthy non-clinical populations, not taking ASD diagnosis into account.

The results of the abovementioned studies pushed researchers to uncover the relationships between other $O X T r$ variants in the promoter region and ASD in greater depth. Among these, rs2268493 has been found to correlate with ASD diagnosis (Yrigollen et al., 2008; Di Napoli et al., 2014), specifically with social withdrawal and repetitive behavior, both as an individual SNP and as part of a haplotype (Ebstein et al., 2009; Francis et al., 2016). There are also evidences of its relation with social cognitive performances in patients with schizophrenia (Davis et al., 2014) and in children diagnosed with ADHD (Ayaz et al., 2015); both disorders share features and symptoms with ASD. Campbell and colleagues found a statistically significant correlation of rs2268493 with autism but the significance was not retained after multiple comparison analysis (Campbell et al., 2011).

In the $3^{\prime}$-untranslated region of the OXTr gene, there is another SNP investigated in association with ASD. rs1042778 was found to be statistically correlated to ASD diagnosis in Caucasian and Jewish populations (Jacob et al., 2007; Lerer et al., 2008; Campbell et al., 2011), but the same result was not confirmed in the Japanese population (Liu et al., 2010). Furthermore, there are suggestions of its implication in aggression (Israel et al., 2009; Johansson et al., 2012; Malik et al., 2012) and prosocial behavior (Israel et al., 2009). With respect to rs13316193, there have been a few studies that found an association of this SNP, contained in a haplotype, with ASD (Ebstein et al., 2009; Liu et al., 2010). As an individual SNP, it has been associated with poor social abilities (Ebstein et al., 2012). Other SNPs have been tested in the last decade, but results are still few and contradictory (Apicella et al., 2010; Tansey et al., 2010), suggesting that these data need to be interpreted with caution and that more research is necessary. Other variants of OXTr that have been found to be related to autism and autistic traits are rs237897 (Lerer et al., 2008; Yrigollen et al., 2008; Wu et al., 2012; Kranz et al., 2016), rs237889 and rs2268494 (Lerer et al., 2008), rs237887 and rs237885 (Liu et al., 2010). Campbell and colleagues found a correlation between rs7632287 and multiple domains of ASD (Campbell et al., 2011) and childhood social problems such as pair-bonding between mother and child (Walum et al., 2012).

\section{AVPR SNPs and Autism}

Arginine-vasopressin is involved in regulatory mechanisms of social behavior like attachment, aggressive behavior and anxiety due to the activity of $A V P$ receptors in the hypothalamicpituitary-adrenal axis (Tansey et al., 2011). In a recent study, Shou and colleagues found that children with ASD, as compared to typically developing ones, showed a positive correlation between $A V P$ levels in the plasma, increased volume in the left amygdala and left hippocampus, and decreased volume in the bilateral hypothalamus, suggesting a contribution of these structural and functional changes to the etiology of ASD (Shou et al., 2017). Studies about social behavior have shown the involvement of AVPR1a and AVPR1b gene polymorphisms (Avinun et al., 2011; Zai et al., 2012). Unlike oxytocin receptor gene, argininevasopressin receptor is composed by different encoder genes, such as AVPR1a, AVPR1b and AVPR2. In particular, two nucleotides located in the $5^{\prime}$ promoter region of $A V P r$; RS1 and RS3, have been the focus of research in this field, with evidence showing that these microsatellites modulate a wide range of social behaviors in both ASD and neurotypical populations (Kim et al., 2002; Wassink et al., 2004; Francis et al., 2016).

Arginine vasopressin receptor la $(A V P R 1 a)$ is largely expressed throughout the brain (Kantojärvi et al., 2015), but also in the liver, kidneys, and vasculature. It is considered to be a crucial receptor for regulation of a set of social behaviors in humans (Ebstein et al., 2009, 2012; Francis et al., 2016), such as altruism and social integration (Israel et al., 2008; Knafo et al., 2008), prepulse inhibition (Levin et al., 2009), emotional and cognitive empathy (Israel et al., 2008; Knafo et al., 2008; Moons et al., 2014; Uzefovsky et al., 2015; Wang et al., 2016), processing and recognition of facial expressions (Israel et al., 2008; Knafo et al., 2008; Moons et al., 2014; Uzefovsky et al., 2015; Wang et al., 2016) and non-human mammals (Murakami et al., 2011; Freeman et al., 2014; Paré et al., 2016; Lesse et al., 2017). Even though vasopressin distribution patterns are quite similar across species, AVPR1a partitions differ both between and within species. In humans, vasopressin production occurs in the hypothalamus, bed nucleus of the stria terminalis, and the 


\section{Oxytocin receptor gene single nucleotide polymorphisms schema}

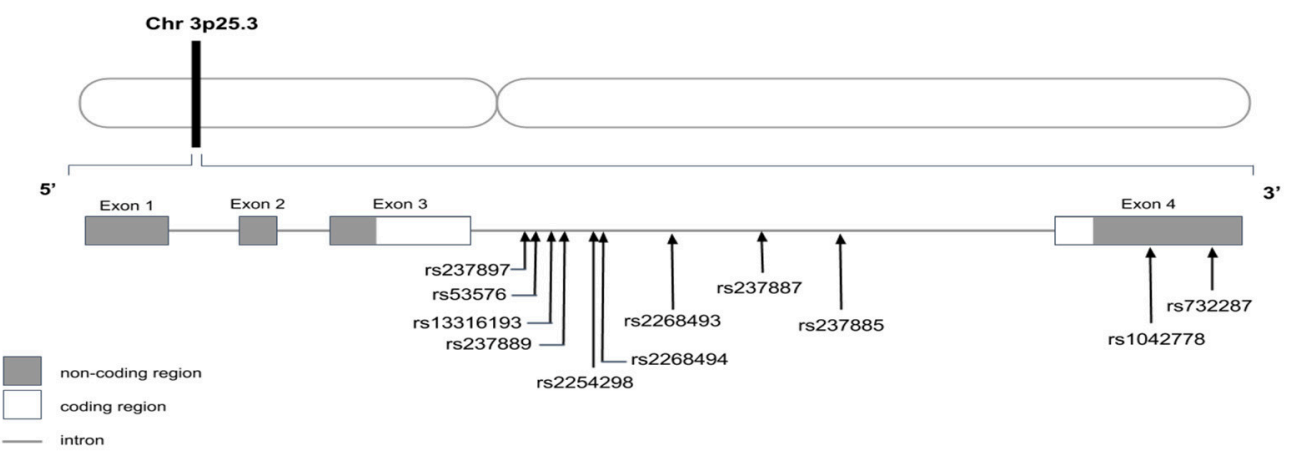

FIGURE 1 | Oxytocin receptor gene schema with location of discussed single nucleotides polymorphisms.

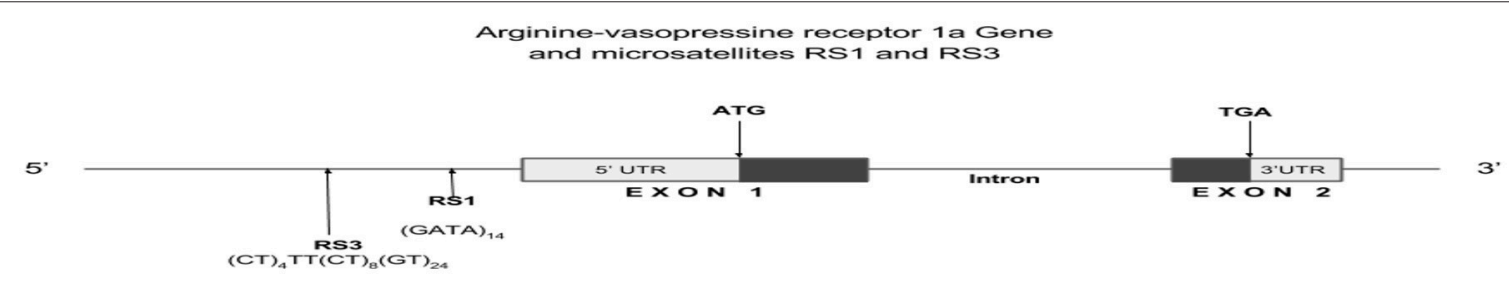

FIGURE 2 | Arginine-vasopressin receptor 1a gene schema with location of microsatellites RS1 and RS3.

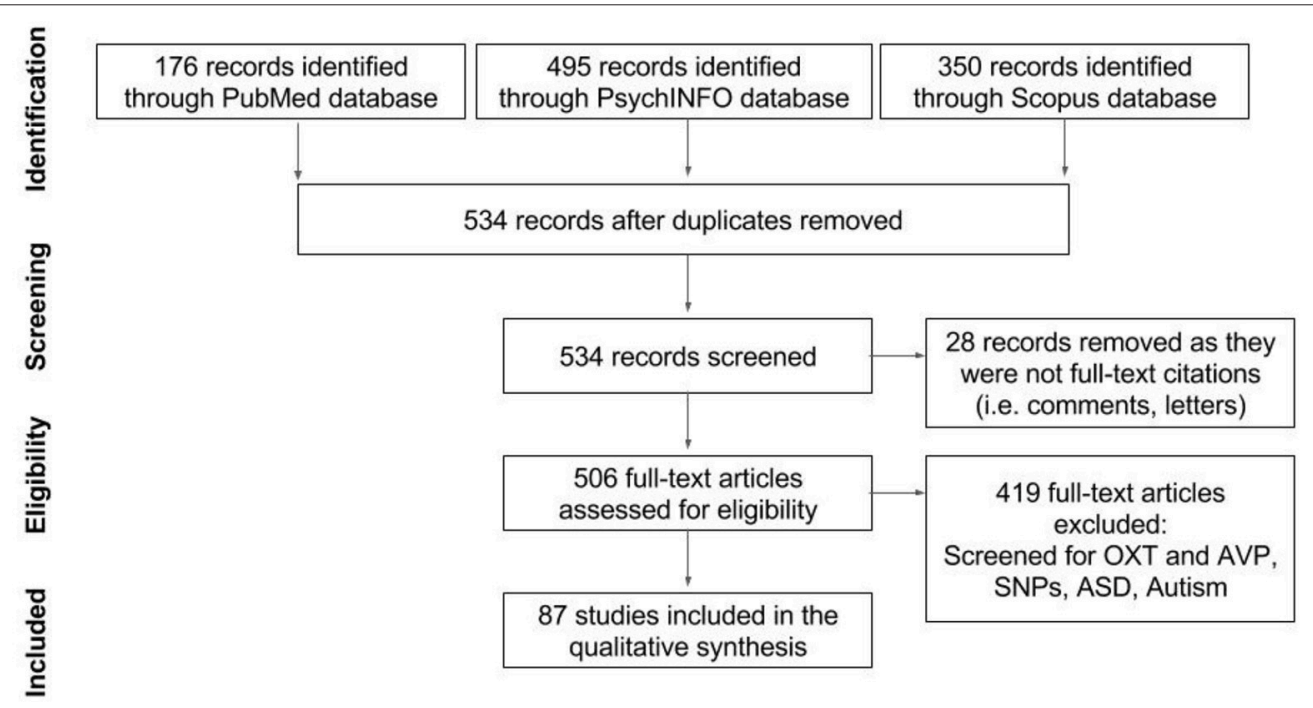

Database screening conducted in December 2017

FIGURE 3 | Flowchart of the screening process for articles to be included in qualitative analysis. 
TABLE 1 | Articles included in the review, with SNPs involved, sample size, and $p$-values.

\begin{tabular}{|c|c|c|c|c|}
\hline Manuscript & Topic & Gene/SNP involved & Sample size & $p$-value \\
\hline Abrahams and Geschwind, 2008 & Neurobiology of ASD & OXTr - AVPR1a & Review article & - \\
\hline Apicella et al., 2010 & Social preferences & $\begin{array}{l}\text { OXTr rs75775 } \\
\text { OXTr rs2268493 } \\
\text { OXTr rs1042778 }\end{array}$ & 684 participants & $>0.05$ \\
\hline Auer et al., 2015 & Rejection sensitivity & OXTr rs53576 & 94 participants & 0.036 \\
\hline Avinun et al., 2012 & Maternal behavior & AVPR1a RS3 & 252 dyads (mother - twins) & $<0.01$ \\
\hline Avinun et al., 2011 & Altruistic behavior & AVPR1a RS3 & 158 twins & 0.04 \\
\hline $\begin{array}{l}\text { Bakermans-Kranenburg and van } \\
\text { ljzendoorn, } 2014\end{array}$ & Social behavior & $\begin{array}{l}\text { OXTr rs53576 } \\
\text { OXTr rs2254298 }\end{array}$ & $\begin{array}{l}17557 \text { participants } \\
13452 \text { participants }\end{array}$ & $\begin{array}{l}>0.05 \\
>0.05\end{array}$ \\
\hline Bielsky and Young, 2004 & Social recognition & $\begin{array}{l}\text { AVPR1a } \\
\text { AVPR1b } \\
\text { OXTr }\end{array}$ & Review article & - \\
\hline Bielsky et al., 2005 & Social recognition & AVPR1a & Mice & $<0.01$ \\
\hline Brüne, 2012 & Psychopathology & OXTr rs2254298 & Review article & - \\
\hline Caldwell et al., 2010 & Social dominance & AVPR1b & Mice & $<0.04$ \\
\hline Caldwell et al., 2008 & Aggression & AVPR1b & Review article & - \\
\hline Campbell et al., 2011 & Association study & $\begin{array}{l}\text { OXTr2268493 } \\
\text { OXTr rs1042778 } \\
\text { OXTr rs7632287 }\end{array}$ & 2333 ASD & $\begin{array}{l}0.043 \\
0.037 \\
0.016\end{array}$ \\
\hline Chakrabarti et al., 2009 & Autistic traits & $\begin{array}{l}\text { OXTr SNPs } \\
\text { AVPR1a } \\
\text { AVPR1b }\end{array}$ & 349 participants & $\begin{array}{l}>0.05 \\
>0.05 \\
>0.05\end{array}$ \\
\hline Chang et al., 2014 & Social integration & $\begin{array}{l}\text { OXTr rs53576 } \\
\text { AVPR1a }\end{array}$ & $>11.000$ individuals & $\begin{array}{l}0.02 \\
>0.05\end{array}$ \\
\hline Chen et al., 2011 & Social support and stress & $\begin{array}{l}\text { OXTr rs53576 } \\
\text { OXTr rs2268493 }\end{array}$ & 194 participants & $\begin{array}{l}<0.01 \\
0.00637\end{array}$ \\
\hline Di Napoli et al., 2014 & Autistic traits and Asperger & $\begin{array}{l}\text { OXTr rs53576 } \\
\text { OXTr rs2254298 }\end{array}$ & 530 individuals & $\begin{array}{l}0.1287 \\
0.0802\end{array}$ \\
\hline Domes et al., 2007 & "Mind-reading" & $\mathrm{OXT}$ & 30 participants & $<0.006$ \\
\hline Donaldson and Young, 2008 & Social behavior & $\begin{array}{l}\text { OXT } \\
\text { AVP }\end{array}$ & Review article & - \\
\hline Ebstein et al., 2009 & Social behavior & $\begin{array}{l}\text { OXTr } \\
\text { AVPR1a }\end{array}$ & Review article & - \\
\hline Ebstein et al., 2012 & Social behavior & $\begin{array}{l}\text { OXT, OXTr } \\
\text { AVP, AVPR1a }\end{array}$ & Review article & - \\
\hline Francis et al., 2016 & Association with ASD & $\begin{array}{l}\text { OXTr rs2268493 } \\
\text { OXTr rs2254298-rs2268493 } \\
\text { AVPR1a RS1-RS3 } \\
\text { AVPR1b rs28632197 } \\
\text { AVPR1b rs35369693 }\end{array}$ & 〜200 families & $\begin{array}{l}0.05 \\
0.026 \\
<0.05 \\
0.005 \\
0.025\end{array}$ \\
\hline Furman et al., 2011 & Amygdala Volume & OXTr rs2254298 & 51 participants & $\begin{array}{l}\text { Left: } 0.040 \\
\text { Right: } 0.036\end{array}$ \\
\hline Gregory et al., 2009 & OXTr deficiency in ASD & OXTr & 119 probands & 0.0389 \\
\hline Guastella and MacLeod, 2012 & Review article & OXT & - & - \\
\hline Guoynes et al., 2017 & Intranasal OXT effects & $\begin{array}{l}\text { OXT } \\
\text { AVP }\end{array}$ & 173 prairie voles & $\begin{array}{l}\text { PVN: }<0.05 \\
\text { SON: }<0.01\end{array}$ \\
\hline Hammock and Young, 2002 & Variation in social behavior & AVPR1a & Review article & - \\
\hline Hammock and Young, 2006 & Pair bonding - ASD & $\begin{array}{l}\text { OXT } \\
\text { AVPR1a }\end{array}$ & Review article & - \\
\hline Haram et al., 2015 & Emotional withdrawal & OXTr rs53576 & 1154 participants & 0.007 \\
\hline Harony and Wagner, 2010 & Social behavior and ASD & $\begin{array}{l}\text { OXT } \\
\text { AVP }\end{array}$ & Review article & - \\
\hline Heinrichs and Domes, 2008 & Social behavior & $\begin{array}{l}\text { OXT } \\
\text { AVP }\end{array}$ & Review article & - \\
\hline Inoue et al., 2010 & Amygdala volume & OXTr2254298 & 208 participants & 0.004 \\
\hline
\end{tabular}


TABLE 1 | Continued

\begin{tabular}{|c|c|c|c|c|}
\hline Manuscript & Topic & Gene/SNP involved & Sample size & $p$-value \\
\hline Insel et al., 1999 & Oxytocin, vasopressin and ASD & $\begin{array}{l}\text { OXT } \\
\text { AVP }\end{array}$ & Review article & - \\
\hline Israel et al., 2008 & Autism and altruism & $\begin{array}{l}\text { OXTr } \\
\text { AVPR1a RS3 }\end{array}$ & 203 participants & $<0.05$ \\
\hline Israel et al., 2009 & Prosocial behavior & OXTr rs1042778 & 203 participants & 0.001 \\
\hline Jacob et al., 2007 & Association study & $\begin{array}{l}\text { OXTr rs53576 } \\
\text { OXTr rs2254298 }\end{array}$ & 57 trios & $\begin{array}{l}0.76 \\
0.03\end{array}$ \\
\hline Kantojärvi et al., 2015 & Association study & AVPR1a RS1 & 205 families & 0.049 \\
\hline Kim et al., 2002 & $\begin{array}{l}\text { AVPR1a transmission } \\
\text { disequlibrium in autism }\end{array}$ & AVPR1a RS1 & 115 trios & 0.023 \\
\hline Lerer et al., 2008 & Association study & $\begin{array}{l}\text { OXTr rs2268494 } \\
\text { OXTr rs1042778 }\end{array}$ & 152 subjects & $\begin{array}{l}0.0117 \\
0.014\end{array}$ \\
\hline Lim et al., 2005 & Animal model of autism & AVPR1a & Review article & - \\
\hline Liu et al., 2010 & Association study & $\begin{array}{l}\text { OXTr rs53576 } \\
\text { OXTr rs2254298 }\end{array}$ & 217 families & $\begin{array}{l}0.053 \\
0.023\end{array}$ \\
\hline LoParo and Waldman, 2015 & Association study & OXTr rs2254298 & 3941 individuals & 0.0476 \\
\hline Marusak et al., 2015 & $\begin{array}{l}\text { Amygdala responses to social } \\
\text { cues }\end{array}$ & OXTr rs2254298 & 55 participants & 0.048 \\
\hline Meyer-Lindenberg and Tost, 2012 & $\begin{array}{l}\text { Amygdala activation and } \\
\text { personality traits }\end{array}$ & $\begin{array}{l}\text { AVPR1a RS1 } \\
\text { AVPR1a RS3 }\end{array}$ & 228 participants & $\begin{array}{l}<0.03 \\
-\end{array}$ \\
\hline Miller M. et al., 2013 & Sex differences in autism & $\begin{array}{l}\text { OXT } \\
\text { AVP }\end{array}$ & 75 participants & Male: 0.03 \\
\hline Montag et al., 2017 & Association study & OXTr rs2268498 & 817 students & $<0.05$ \\
\hline Moons et al., 2014 & Emotional reaction to stress & $\begin{array}{l}\text { OXTr rs53576 } \\
\text { AVPR1a RS1 }\end{array}$ & 166 participants & $\begin{array}{l}\text { Female: } 0.39 \\
\text { Male: } 0.012\end{array}$ \\
\hline Nyffeler et al., 2014 & Association study & $\begin{array}{l}\text { OXTr rs53576 } \\
\text { OXTr rs2254298 } \\
\text { OXTr rs2268494 }\end{array}$ & 253 participants & $\begin{array}{l}- \\
0.005 \\
0.005\end{array}$ \\
\hline Parker et al., 2014 & Social impairment prediction & $\begin{array}{l}\text { OXTr rs53576 } \\
\text { OXTr rs2254298 }\end{array}$ & 193 children & $\begin{array}{l}>0.05 \\
0.0292\end{array}$ \\
\hline Peñagarikano, 2017 & Animal model of autism & OXT & Review article & - \\
\hline Poulin et al., 2012 & Proscial behavior & $\begin{array}{l}\text { OXTr rs53576 } \\
\text { AVPR1a RS1 } \\
\text { AVPR1a RS3 }\end{array}$ & 348 participants & $\begin{array}{l}<0.001 \\
<0.001 \\
-\end{array}$ \\
\hline Procyshyn et al., 2016 & Association study & $\begin{array}{l}\text { AVPR1a RS1 } \\
\text { AVPR1a RS3 }\end{array}$ & 873 participants & $\begin{array}{l}- \\
0.0419\end{array}$ \\
\hline Rijlaarsdam et al., 2017 & Association study & OXTr rs53576 & 746 children & $<0.001$ \\
\hline Rodrigues et al., 2009 & Empathy and stress reactivity & OXTr rs53576 & 192 participants & $>0.05$ \\
\hline Sala et al., 2011 & Animal model of autism & OXTr & Mice & $<0.01$ \\
\hline Saphire-Bernstein et al., 2011 & Psychological resources & OXTr rs53576 & 261 participants & 0.002 \\
\hline Schneider-Hassloff et al., 2016 & $\mathrm{G}^{\star} \mathrm{E}$ interaction and mentalizing & OXTr rs53576 & 195 participants & $\begin{array}{l}\text { Amygdala voulme: } \\
p=0.034\end{array}$ \\
\hline Shou et al., 2017 & $\begin{array}{l}\text { AVP pathways in ASD (fMRI } \\
\text { study) }\end{array}$ & AVP & 14 ASD children & $\begin{array}{l}\text { IAmygd - rSMG: } \\
p=0.02\end{array}$ \\
\hline Stoop, 2012 & Neuromodulation & $\begin{array}{l}\text { OXT } \\
\text { AVP }\end{array}$ & Review article & - \\
\hline Tansey et al., 2010 & Association study & OXTr SNPS (18) & 436 ASD subjects & $>0.05$ \\
\hline Tansey et al., 2011 & Functionality of AVPR1a & $\begin{array}{l}\text { AVPR1a rs11174815 } \\
\text { AVPR1a RS1 }\end{array}$ & 177 families & $\begin{array}{l}0.008 \\
0.036\end{array}$ \\
\hline Thompson et al., 2007 & Prosocial effects & OXTr & Mice & $<0.01$ \\
\hline Tost et al., 2010 & $\begin{array}{l}\text { Prosocial effects and brain } \\
\text { structure }\end{array}$ & OXTr rs53576 & 212 participants & Brain struct: 0.047 \\
\hline & & & & Prosociality: 0.05 \\
\hline Tseng et al., 2017 & Breastfeeding and ASD & OXT & >1000 ASD children & 0.002 \\
\hline Uzefovsky et al., 2015 & Emotional and cognitive empathy & $\begin{array}{l}\text { OXTr rs53576 } \\
\text { AVPR1a RS3 }\end{array}$ & 367 participants & $\begin{array}{l}0.029 \\
0.002\end{array}$ \\
\hline
\end{tabular}


TABLE 1 | Continued

\begin{tabular}{|c|c|c|c|c|}
\hline Manuscript & Topic & Gene/SNP involved & Sample size & $p$-value \\
\hline Veenema and Neumann, 2008 & $\begin{array}{l}\text { Regulation of complex social } \\
\text { behavior }\end{array}$ & $\begin{array}{l}\text { OXT } \\
\text { AVP }\end{array}$ & Mice & $<0.05$ \\
\hline Veenema et al., 2010 & Male aggression & AVP & Mice & $<0.05$ \\
\hline Verhallen et al., 2017 & Face recognition & rs237887 & 370 participants & 0.027 \\
\hline Walum et al., 2012 & Social behavior & $\begin{array}{l}\text { OXTr rs53576 } \\
\text { OXTr rs2254298 }\end{array}$ & 40 participants & 0.008 \\
\hline Wassink et al., 2004 & Association study & AVPR1a & 190 ASD subjects & 0.02 \\
\hline Watanabe et al., 2017 & Behavioral response in ASD & $\begin{array}{l}\text { OXTr rs53576 } \\
\text { OXTr rs2254298 }\end{array}$ & 38 ASD subjects & $\begin{array}{l}0.009 \\
0.0009\end{array}$ \\
\hline Weisman et al., 2015 & Cognitive empathy & OXTr rs53576 & 1463 participants & $<0.05$ \\
\hline Wermter et al., 2010 & Association study & $\begin{array}{l}\text { OXTr rs53576 } \\
\text { OXTr rs2254298 }\end{array}$ & 100 ASD subjects & $\begin{array}{l}0.22 \\
1.0\end{array}$ \\
\hline Wersinger et al., 2004 & Social motivation & AVPR1b & Mice & $<0.05$ \\
\hline Winslow and Insel, 2004 & Social recognition & $\begin{array}{l}\text { AVPR1a } \\
\text { AVPR1b }\end{array}$ & Review article & - \\
\hline Wu et al., 2012 & Empathy & OXTr rs2254298 & 101 participants & $<0.05$ \\
\hline Wu et al., 2015 & Prosociality & AVPR1b rs28373064 & 256 participants & 0.048 \\
\hline Wu et al., 2005 & Association study & $\begin{array}{l}\text { OXTr rs53576 } \\
\text { OXTr rs2254298 }\end{array}$ & 195 ASD families & $\begin{array}{l}0.0101 \\
0.0222\end{array}$ \\
\hline Yamasue, 2013 & Autistic social behavior & $\begin{array}{l}\text { OXTr rs53576 } \\
\text { OXTr rs2254298 }\end{array}$ & Review article & - \\
\hline Yang et al., 2010a & Association study & $\begin{array}{l}\text { AVPR1a RS1 } \\
\text { AVPR1a RS3 }\end{array}$ & 148 trios & $\begin{array}{l}<0.001 \\
<0.001\end{array}$ \\
\hline Yang et al., 2010b & Association study & $\begin{array}{l}\text { AVPR1a rs7294536 } \\
\text { AVPR1a rs10877969 }\end{array}$ & 151 trios & $\begin{array}{l}0.002 \\
<0.001\end{array}$ \\
\hline Yang et al., 2017 & Association study & $\begin{array}{l}\text { AVPR1a RS1 } \\
\text { AVPR1a RS3 } \\
\text { AVPR1a rs7294536 } \\
\text { AVPR1a rs10877969 }\end{array}$ & 212 families & $\begin{array}{l}- \\
0.02 \\
0.039 \\
<0.001\end{array}$ \\
\hline Yirmiya et al., 2006 & Association study & $\begin{array}{l}\text { AVPR1a RS1 } \\
\text { AVPR1a RS3 } \\
\text { AVR }\end{array}$ & 116 families & 0.004 \\
\hline Young, 1999 & Social Behavior & $\begin{array}{l}\text { OXTr } \\
\text { AVPR1a }\end{array}$ & Mice & $\begin{array}{l}<0.05 \\
<0.05\end{array}$ \\
\hline Yrigollen et al., 2008 & Affiliative behavior and ASD & OXTr & 151 families & $<0.05$ \\
\hline Zai et al., 2012 & Child aggression & AVPR1b rs35369693 & 177 children & 0.003 \\
\hline Zhang et al., 2017 & Association with ASD & $\begin{array}{l}\text { OXT } \\
\text { AVP }\end{array}$ & Review article & - \\
\hline Zimmermann et al., 2016 & Social interaction and aggression & OXT & Zebrafish & $<0.05$ \\
\hline
\end{tabular}

medial amygdala, while binding sites are located in the lateral septum, thalamus, basal amygdaloid nucleus, and brainstem (Meyer-Lindenberg et al., 2009; Zhang et al., 2017). Starting from the beginning of the century, many authors highlighted an association between variation at AVPR1a and neuropsychiatric phenotypes, with a wide focus on autism (Kim et al., 2002; Yirmiya et al., 2006; Tansey et al., 2011; Francis et al., 2016), parental behavior (Avinun et al., 2012) and social behavior in general (Yirmiya et al., 2006; Meyer-Lindenberg et al., 2009; Ebstein et al., 2012). Human AVPR1a promoter region contains two microsatellite repeats, which are highly polymorphic in the general population, and have been studied in association with individual variation in social behavior (Ebstein et al., 2012; Procyshyn et al., 2016): RS1, a (GATA)14 repeat sequence, and RS3, a complex (CT)4TT(CT)8(GT)24 repeat sequence (Thibonnier et al., 2000). Studies report an association between repeat number of these microsatellites and expression of AVPR1a in the hippocampus and the brain in general (Knafo et al., 2008). As a consequence, this can affect the modulation of social behavior on non-clinical autistic phenotypes (Carver and Harmon-Jones, 2009; Moons et al., 2014) and autistic traits (Israel et al., 2008; Procyshyn et al., 2016).

Specifically, results revealed an association between "shortalleles" of variant RS1 and a decrease in transcription of AVPR1a gene in the amygdala, leading to poorer prosocial behavior (Poulin et al., 2012; Kantojärvi et al., 2015; Yang et al., 2017) and higher amygdala activation during a facial recognition task (Meyer-Lindenberg et al., 2009). Kim et al. (2002) have been 
the first to conduct molecular genetic studies on AVPR1a and ASD, finding that one of the alleles of RS1 showed increased transmission in a family based study of 115 trios (mother, father, and child). More studies on the same microsatellite highlighted similar results (Wassink et al., 2004; Yirmiya et al., 2006; Yang et al., 2010a; Tansey et al., 2011; Kantojärvi et al., 2015). On the contrary, it is the "long-alleles" variant in RS3 that is associated with an increased in activity of the amygdala, which has been linked to social withdrawal. Studies show associations between this microsatellite and cognitive empathy and social skills in humans (Bosch and Neumann, 2008; Knafo et al., 2008; Harony and Wagner, 2010; Avinun et al., 2011; Uzefovsky et al., 2015), especially in the context of mother-infant play (Avinun et al., 2012). The "long-alleles" variant in RS3 has been linked to a risk of developing ASD too (Ebstein et al., 2009; Kantojärvi et al., 2015; Procyshyn et al., 2016). These results confirmed previous findings on animal models (Insel et al., 1994).

The studies reviewed show that AVPR1a modulation over brain functioning and ASD traits, with specific involvement in the domains of social communication, emotion and cognition, is often considered a consequence of the haplotype RS1-RS3 (Meyer-Lindenberg et al., 2009). Some authors have started investigating other SNPs belonging to the promoter region of AVPR1a gene to investigate if the etiology of autism might be modulated by a joint mechanism. An Irish study conducted in 2011 tagged four SNPs of the AVPR1a gene (rs3803107, rs1042615, rs3741865, rs11174815) but results showed that only rs11174815 was weakly correlated to autism (Tansey et al., 2011). Another study found a significant correlation of rs7294536, rs3759292, and rs10877969 on autistic phenotypes in trios in a Korean population (Yang et al., 2010b). Findings from these two studies suggest that the underlying actions of these receptors remain elusive, and that results may be influenced by specific cultural environments.

While the relationship between AVPR1a and ASD is relatively consistent, there is still need for further studies on the impact of AVPR1b on the outcome of autism. At the present, our knowledge is limited to the involvement of this receptor in the modulation of neuroendocrine response to stress (Muráni et al., 2010; Roper et al., 2011) and developmental disorders (Dempster et al., 2009; van West et al., 2009). Evidence about the role of $A V P R 1 b$ in social and affiliative behaviors come from mice models (Wersinger et al., 2004; Caldwell et al., 2008, 2010; Pagani et al., 2015). There are few results that shed light on the involvement of $A V P R 1 b$ in autistic traits in humans (Zai et al., 2012; Luppino et al., 2014; Wu et al., 2015), although it is possible to find evidence in literature that associates AVPR $1 b$ rs28632197 with comorbid conditions such as panic disorder (Keck et al., 2008). Exception being a recent report by Francis and colleagues on a correlation between AVPR $1 b$ rs35369693 and autism (Francis et al., 2016).

\section{OXT, AVP and Differences between Sexes}

As already mentioned, ASD is well known to be sexually biased, occurring 4 times more in boys than girls. Studies on animal models, specifically in mammals, showed that the influence of $O X T$ and $A V P$ on brain activity and behavior is sexually-dimorphic, with stronger effects of oxytocin on females and of arginine-vasopressin in males. Hence, oxytocinergic and vasopressinergic systems may be related to the sex-biased occurrence of ASD.

Among the works discussed in the present review, there are few evidences showing sex-specific effects of $O X T r$ and $A V P r$ (and their variations), that enhanced the hypothesis of a sexually dimorphic modulation of autistic spectrum traits, and social behaviors in general, by these two neurotransmitters. Regarding OXTr, a study by Chen and colleagues found a significant sexdependent effect in the interaction between rs2254298 and social functioning. Female A-carriers tended to show greater anxiety while men reported more autism-associated traits (Chen et al., 2011).

Furthermore, research by Chang and colleagues on social connectedness highlighted an opposite association between men and women with respect to rs4686302, and a direct correlation between rs53576 and women only, suggesting that sex can determine differential effects from the same variation (Chang et al., 2014). Other studies revealed sex-based differences in the amygdala, showing greater gray matter volume in male A-carriers for rs53576 (Tost et al., 2010) and a negative correlation with scores in reward tasks (Ebstein et al., 2012). The same amygdalial structural observation was also detected in homozygous G-carrier women for rs2254298 (Furman et al., 2011). Sex has also been shown to predict emotional empathy, with women scoring higher than men (Wu et al., 2012; Uzefovsky et al., 2015), although some studies did not find any differences (Rodrigues et al., 2009).

With regards to diagnosis of $\mathrm{ASD}$, significant correlations were found between the Japanese male population and rs2254298 (Liu et al., 2010; Watanabe et al., 2017) and rs53576 (Watanabe et al., 2017). In the case of DNA methylation, as compared to healthy individuals, Gregory and colleagues found a significant sex-specific decrease in the expression of $O X \operatorname{Tr}$ in males with autism, correlated to an increase in methylation at a specific site. Heterozygous deletion of the same OXTr site in mothers included in the sample did not result in diagnosis of ASD while deletion in males did (Gregory et al., 2009).

Switching to the vasopressin system, a few studies explored sex differences of $A V P, A V P r$ and its variation on autism and social behaviors. Animal models showed that $A V P$ in the central nervous system (CNS) activates brain regions (i.e., amygdala, hyppocampus) that coordinate specific-social behaviors, such as intermale aggression (Veenema et al., 2010); with regards to the receptors, it is possible to find in literature evidence that $A V P R 1 a$ is necessary and sufficient for social recognition (Bielsky et al., 2005), and that deficits are worse in the male sample (Winslow and Insel, 2004). Furthermore, AVP in the CNS coordinates a range of different social behaviors. Rodents, specifically male samples of $A V P R 1 B$ knockout mice, have also been used to elucidate mechanisms related to social dominance (Caldwell et al., 2010).

With regards to humans, prepulse inhibition (PPI) has been studied in both sexes, due to its involvement in social skills deficits. In 2009, two studies explicated the correlation between PPI and AVPR1a RS3, finding higher responses in long-carriers 
(Levin et al., 2009), which were even more evident in men (Ebstein et al., 2009). As for AVPR1B, rs28373064 was found to be more linked to prosociality in men compared to women (Wu et al., 2015) and this mechanism might be modulated by emotional empathy. A study conducted on children with ASD highlighted a strong association between $A V P$ blood plasma levels and the connection between the left amygdala and left supermarginal gyrus, but only in boys (Shou et al., 2017). The amygdala is well-known to be a sexually-dimorphic major nucleus, receiving neurons from the hypothalamus that contains $A V P$, so this might shed light on mechanisms that contribute to the etiology of ASD.

With regards to separate analysis by sex, some incongruence is still present in literature (Knafo et al., 2008), underlining the need for lower variability and bigger sample sizes (Procyshyn et al., 2016). The included studies represent only a small portion of the evidences that underlie the sexually-dymorphic nature of OXT and AVP in the etiology of autism. Looking at these neurotransmitters together, a study by Miller and colleagues in an ASD population found higher levels of OXT in blood plasma in girls, and of $A V P$ in boys; this difference was also reflected in a diverse association with autistic symptoms, especially with anxiety levels and repetitive behaviors (Miller M. et al., 2013). OXTr and AVPr genes have been found to interact with blood plasma levels of oxytocin and vasopressin in generating responses to emotional stress and these effects are displayed differently in men and women (Moons et al., 2014).

\section{DISCUSSION}

Since the early period of the animal kingdom, oxytocin has prevailed as a modulator of social affiliative behaviors across species of mammals, birds and amphibians alike (Gimpl and Fahrenholz, 2001; Donaldson and Young, 2008). Thereafter, came the emergence of a second integral neuropeptide which we have come to know as arginine-vasopressin, along with their respective receptors (Gimpl and Fahrenholz, 2001; Donaldson and Young, 2008). Like oxytocin, cells of the PVN and supraoptic nuclei of the hypothalamus express vasopressin too, conferring these two neuropeptides to a series of common features, starting from their parallel genetic structures, and eventually, similarities in how both these molecules exert their effects on the same neural structures in the central and autonomic nervous systems (Zhang et al., 2017). Being agonists, these two molecules are able to mutually bind to the other's receptors, influencing one another's functions. Although they share many common features, some differences between them do exist. For instance, OXT possesses a single receptor type, while $A V P$ was found to have three different subtypes ( $A V P R 1 a, A V P R 1 b, A V P R 2)$. With regard to implication in social behaviors, $A V P$ has been associated with pair-bond formation in monogamous species (Tickerhoof and Smith, 2017), while evidences in literature suggest that $O X T$ is more broadly related to prosocial functioning. Furthermore, these two neuropeptides and their receptor variants exert different sexdependent effects during physiological responses to stressors (Moons et al., 2014), suggesting an intriguing topic that still needs to be explored in-depth.
Beyond continuously developing knowledge about how $\mathrm{OXTr}$ and $A V P r$ influence behaviors, researchers of today have devoted much attention to the roles these receptors play in the etiology of sex-biased neuropsychiatric disorders involving impairments on a social level, such as autism (Knapp et al., 2009). Indeed, numerous studies have been conducted to further uncover the effects of these neuropeptides in both sexes, revealing some sex-related differences on the "social behavior neural network" in clinical and non-clinical populations (Taylor et al., 2010; Dumais and Veenema, 2016; DiBenedictis et al., 2017). Directing resources toward the OXT-AVP system may potentially lead us to uncover critical biomarkers of ASD, allowing for earlier diagnosis, intervention and ultimately a more promising prognosis. An array of cutting-edge genetic tools, including highthroughput SNP sequencing, array-based copy number variant analysis, as well as genome-wide expression profiling, have surfaced in response to this need. These powerful molecular approaches, in combination with methods in neuroscience (i.e., imaging) and biochemistry (i.e., intranasal administration of $O X T)$, pave the way for translational research that taps upon $O X T-A V P$ pathways.

\section{CONCLUSION AND FUTURE RESEARCH}

In this review, we have systematically examined the existing literature and have highlighted key SNPs of OXTr and AVPr that have been associated to ASD. The massive number of papers obtained from our search process illustrated how keen the scientific community is in endeavoring to converge disparate information from various fields (such as neurobiology, genetics, psychiatry, neuroscience, physiology, pharmacology, endocrinology, immunology) so as to generate new knowledge on ASD that can propel the development of novel preventive and intervention solutions.

OXTr and $A V P r$ have been widely studied, being strongly related to social behavior. Specifically, RS1 and RS3, polymorphisms contained within the promoter region of AVPR1, and rs28632197 and rs35369693 SNPs of AVPR1b, have been found to be significantly linked to ASD. OXTr remains a key contributor as well, with rs53576 and rs225429 SNPs consistently shown to be associated with ASD. Controversy within the literature due to inconsistent findings have been discussed, including the varying effects of these polymorphisms on different ethnic groups, lack of agreement on which allele confers vulnerability for ASD, and even contention regarding the involvement of these SNPs in the first place. These discrepancies stem from inconsistent methodology, such as non-standardized inclusion criteria, sampling, data collection and analysis, and possibly requires systematic replication to be reconciled.

While much has been gleaned from studies investigating SNPs of OXTr and AVPr, the mechanisms behind how these polymorphisms contribute to ASD have yet to be fully elucidated. Three main suggestions for future studies can be generated from this systematic review. Firstly, given the male-biased nature of ASD, more emphasis should be placed on fully elucidating the differential mechanisms of $A V P r$ and $O X T r$ in males and females. Since sex-dependent differences in expression of these 
genes have been observed (Miller M. et al., 2013; Dumais and Veenema, 2016), this is a potential direction in which the field of genetics can take. However, the discrepancy in the prevalence between sexes in ASD in clinical populations might make this aim hard to achieve. Secondly, given the intricately similar characteristics and shared mechanisms of AVP and $O X T$ on their receptor classes, additional studies should be conducted to uncover the interplay of these molecules at the genetic (i.e., polymorphisms of receptors), physiological (i.e., co-regulation of physiological pathways) and systemic levels (i.e., dual modulation of brain structures) in contributing to ASD development. Lastly, given the vastly contrasting outlook of DSM-5 from previous DSM manuals, with the former adopting the concept of "spectrum," future meta-analyses should differentiate between the use of these manuals in ASD diagnosis.

More importantly, the story behind the development of autism has not been fully told until environmental influences have been investigated in conjunction with functional molecular analyses. It is largely plausible that $O X T r$ and $A V P r$ expression is more complex, regulated and fine-tuned at the cross-roads of cis-acting (i.e., SNPs) and trans-acting factors such as temporal

\section{REFERENCES}

Abrahams, B. S., and Geschwind, D. H. (2008). Advances in autism genetics: on the threshold of a new neurobiology. Nat. Rev. Genet. 9, 341-355. doi: $10.1038 / \mathrm{nrg} 2346$

American Psychiatric Association (2013). Diagnostic and Statistical Manual of Mental Disorders (DSM-5 $\left.{ }^{\circledR}\right)$. American Psychiatric Pub.

Apicella, C. L., Cesarini, D., Johannesson, M., Dawes, C. T., Lichtenstein, P., Wallace, B., et al. (2010). No association between oxytocin receptor (OXTR) gene polymorphisms and experimentally elicited social preferences. PLoS ONE 5:e11153. doi: 10.1371/journal.pone.0011153

Ashwood, P., Krakowiak, P., Hertz-Picciotto, I., Hansen, R., Pessah, I., and Van de Water, J. (2011). Elevated plasma cytokines in autism spectrum disorders provide evidence of immune dysfunction and are associated with impaired behavioral outcome. Brain Behav. Immun. 25, 40-45. doi: 10.1016/j.bbi.2010.08.003

Ashwood, P., Wills, S., and Van de Water, J. (2006). The immune response in autism: a new frontier for autism research. J. Leukoc. Biol. 80, 1-15. doi: $10.1189 /$ jlb. 1205707

Atladóttir, H. O., Thorsen, P., Østergaard, L., Schendel, D. E., Lemcke, S., Abdallah, M., et al. (2010). Maternal infection requiring hospitalization during pregnancy and autism spectrum disorders. J. Autism Dev. Disord. 40, 1423-1430. doi: 10.1007/s10803-010-1006-y

Auer, B. J., Byrd-Craven, J., Grant, D. M., and Granger, D. A. (2015). Common oxytocin receptor gene variant interacts with rejection sensitivity to influence cortisol reactivity during negative evaluation. Horm. Behav. 75, 64-69. doi: 10.1016/j.yhbeh.2015.07.023

Avinun, R., Ebstein, R. P., and Knafo, A. (2012). Human maternal behaviour is associated with arginine vasopressin receptor 1A gene. Biol. Lett. 8, 894-896. doi: 10.1098/rsbl.2012.0492

Avinun, R., Israel, S., Shalev, I., Gritsenko, I., Bornstein, G., Ebstein, R. P., et al. (2011). AVPR1A variant associated with preschoolers' lower altruistic behavior. PLoS ONE 6:e25274. doi: 10.1371/journal.pone. 0025274

Avraham, Y., Berry, E. M., Donskoy, M., Ahmad, W. A., Vorobiev, L., Albeck, A., et al. (2017). Beta-carotene as a novel therapy for the treatment of "Autistic like behavior" in animal models of Autism. Behav. Brain Res. doi: 10.1016/j.bbr.2017.09.041. [Epub ahead of print]. and contextual variables (Carter, 2007). Even though replication studies have been conducted in the past few years, filling this gap in the literature, we have only uncovered the prodigious tip of the iceberg, and simultaneous effort across various research groups, from genetic through to the behavioral levels, is required to fully leverage upon the knowledge we have on $O X T r$ and $A V P r$ to generate effective diagnostic and therapeutic intervention for ASD.

\section{AUTHOR CONTRIBUTIONS}

IC, AA, GE: Conceived, designed, and performed the review, interpreted literature, wrote the paper; and edited and reviewed the papers.

\section{ACKNOWLEDGMENTS}

This study was supported by the scholarship "The relationships between genes, psychological traits and behavior" cofunded by FBK and University of Trento (IC), The Nanyang President Graduate Scholarship (AA) as well as the NAP-SUG program of the Nanyang Technological University (GE).

Ayaz, A. B., Karkucak, M., Ayaz, M., Gokce, S., Kayan, E., Güler, E. E., et al. (2015). Oxytocin system social function impacts in children with attentiondeficit/hyperactivity disorder. Am. J. Med. Genet. Part B Neuropsych. Genet. 168, 609-616. doi: 10.1002/ajmg.b.32343

Bakermans-Kranenburg, M. J., and van Ijzendoorn, M. H. (2014). A sociability gene? Meta-analysis of oxytocin receptor genotype effects in humans. Psychiatr. Genet. 24, 45-51. doi: 10.1097/YPG.0b013e3283643684

Baranek, G. T., Boyd, B. A., Poe, M. D., David, F. J., and Watson, L. R. (2007). Hyperresponsive sensory patterns in young children with autism, developmental delay, and typical development. Am. J. Mental Retard. 112, 233-245. doi: 10.1352/0895-8017(2007)112[233:HSPIYC]2.0.CO;2

Baron-Cohen, S., Knickmeyer, R. C., and Belmonte, M. K. (2005). Sex differences in the brain: implications for explaining autism. Science 310, 819-823. doi: 10.1126/science. 1115455

Belzung, C., and Lemoine, M. (2011). Criteria of validity for animal models of psychiatric disorders: focus on anxiety disorders and depression. Biol. Mood Anxiety Disord. 1:9. doi: 10.1186/2045-5380-1-9

Berko, E. R., Suzuki, M., Beren, F., Lemetre, C., Alaimo, C. M., Calder, R. B., et al. (2014). Mosaic epigenetic dysregulation of ectodermal cells in autism spectrum disorder. PLoS Genet. 10:e1004402. doi: 10.1371/journal.pgen.10 04402

Betancur, C. (2011). Etiological heterogeneity in autism spectrum disorders: more than 100 genetic and genomic disorders and still counting. Brain Res. 1380, 42-77. doi: 10.1016/j.brainres.2010.11.078

Bielsky, I. F., Hu, S. B., Ren, X., Terwilliger, E. F., and Young, L. J. (2005). The V1a vasopressin receptor is necessary and sufficient for normal social recognition: a gene replacement study. Neuron 47, 503-513. doi: 10.1016/j.neuron.2005.06.031

Bielsky, I. F., and Young, L. J. (2004). Oxytocin, vasopressin, and social recognition in mammals. Peptides 25, 1565-1574. doi: 10.1016/j.peptides.2004.05.019

Bittencourt Jacondino, C., Jacondino, C. B., Borges, C. A., and Gottlieb, M. G. (2014). Associação dos polimorfismos rs53576 e rs2254298 do gene receptor da ocitocina com depressão: uma revisão sistemática. Sci. Med. 24:411. doi: 10.15448/1980-6108.2014.4.18173

Bodden, C., van den Hove, D., Lesch, K. P., and Sachser, N. (2017). Impact of varying social experiences during life history on behaviour, gene expression, and vasopressin receptor gene methylation in mice. Sci. Rep. 7:8719. doi: 10.1038/s41598-017-09292-0 
Boksa, P. (2010). Effects of prenatal infection on brain development and behavior: a review of findings from animal models. Brain Behav. Immun. 24, 881-897. doi: 10.1016/j.bbi.2010.03.005

Brown, A. S., and Derkits, E. J. (2010). Prenatal infection and schizophrenia: a review of epidemiologic and translational studies. Am. J. Psychiatry 167, 261-280. doi: 10.1176/appi.ajp.2009.09030361

Bosch, O. J., and Neumann, I. D. (2008). Brain vasopressin is an important regulator of maternal behavior independent of dams' trait anxiety. Proc. Natl. Acad. Sci. U.S.A. 105, 17139-17144. doi: 10.1073/pnas.0807412105

Boyd, B. A., Baranek, G. T., Sideris, J., Poe, M. D., Watson, L. R., Patten, E., et al. (2010). Sensory features and repetitive behaviors in children with autism and developmental delays. Autism Res. 3, 78-87. doi: 10.1002/aur.124

Bralten, J., van Hulzen, K. J., Martens, M. B., Galesloot, T. E., Arias Vasquez, A., Kiemeney, L. A., et al. (2017). Autism spectrum disorders and autistic traits share genetics and biology. Mol. Psychiatry doi: 10.1038/mp.2017.127. [Epub ahead of print].

Brüne, M. (2012). Does the oxytocin receptor (OXTR) polymorphism (rs2254298) confer "vulnerability" for psychopathology or "differential susceptibility"? Insights from evolution. BMC Med. 10:38. doi: 10.1186/1741-7015-10-38

Burket, J. A., Herndon, A. L., Winebarger, E. E., Jacome, L. F., and Deutsch, S. I. (2011). Complex effects of mGluR5 antagonism on sociability and stereotypic behaviors in mice: possible implications for the pharmacotherapy of autism spectrum disorders. Brain Res. Bull. 86, 152-158. doi: 10.1016/j.brainresbull.2011.08.001

Buxbaum, J. D. (2009). Multiple rare variants in the etiology of autism spectrum disorders. Dialogues Clin. Neurosci. 11, 35-43.

Caldwell, H. K., Dike, O. E., Stevenson, E. L., Storck, K., and Young, W. S. III. (2010). Social dominance in male vasopressin $1 \mathrm{~b}$ receptor knockout mice. Horm. Behav. 58, 257-263. doi: 10.1016/j.yhbeh.2010.03.008

Caldwell, H. K., Wersinger, S. R., and Young, W. S. III. (2008). The role of the vasopressin $1 \mathrm{~b}$ receptor in aggression and other social behaviours. Prog. Brain Res. 170, 65-72. doi: 10.1016/S0079-6123(08)00406-8

Campbell, D. B., Datta, D., Jones, S. T., Batey Lee, E., Sutcliffe, J. S., Hammock, E. A., et al. (2011). Association of oxytocin receptor (OXTR) gene variants with multiple phenotype domains of autism spectrum disorder. J. Neurodev. Disord. 3, 101-112. doi: 10.1007/s11689-010-9071-2

Caronna, E. B., Milunsky, J. M., and Tager-Flusberg, H. (2008). Autism spectrum disorders: clinical and research frontiers. Arch. Dis. Child. 93, 518-523. doi: $10.1136 /$ adc. 2006.115337

Carter, C. S. (2007). Sex differences in oxytocin and vasopressin: implications for autism spectrum disorders? Behav. Brain Res. 176, 170-186. doi: 10.1016/j.bbr.2006.08.025

Carver, C. S., and Harmon-Jones, E. (2009). Anger is an approach-related affect: evidence and implications. Psychol. Bull. 135, 183-204. doi: 10.1037/a0013965

Cascio, C., McGlone, F., Folger, S., Tannan, V., Baranek, G., Pelphrey, K. A., et al. (2008). Tactile perception in adults with autism: a multidimensional psychophysical study. J. Autism Dev. Disord. 38, 127-137. doi: $10.1007 / \mathrm{s} 10803-007-0370-8$

Chadman, K. K. (2017). Animal models for autism in 2017 and the consequential implications to drug discovery. Expert Opin. Drug Discov. 12, 1187-1194. doi: 10.1080/17460441.2017.1383982

Chakrabarti, B., Dudbridge, F., Kent, L., Wheelwright, S., Hill-Cawthorne, G., Allison, C., et al. (2009). Genes related to sex steroids, neural growth, and socialemotional behavior are associated with autistic traits, empathy, and Asperger syndrome. Autism Res. 2, 157-177. doi: 10.1002/aur.80

Chakrabarti, S., and Fombonne, E. (2005). Pervasive developmental disorders in preschool children: confirmation of high prevalence. Am. J. Psychiatry 162, 1133-1141. doi: 10.1176/appi.ajp.162.6.1133

Chang, S. C., Glymour, M. M., Rewak, M., Cornelis, M. C., Walter, S., Koenen, K. C., et al. (2014). Are genetic variations in OXTR, AVPR1A, and CD38 genes important to social integration? Results from two large U.S. cohorts. Psychoneuroendocrinology 39, 257-268. doi: 10.1016/j.psyneuen.2013. 09.024

Chang, S. W., Brent, L. J., Adams, G. K., Klein, J. T., Pearson, J. M., Watson, K. K., et al. (2013). Neuroethology of primate social behavior. Proc. Natl. Acad. Sci. U.S.A. 110(Suppl. 2), 10387-10394. doi: 10.1073/pnas.1301213110

Chen, F. S., Kumsta, R., von Dawans, B., Monakhov, M., Ebstein, R. P., and Heinrichs, M. (2011). Common oxytocin receptor gene (OXTR) polymorphism and social support interact to reduce stress in humans. Proc. Natl. Acad. Sci. U.S.A. 108, 19937-19942. doi: 10.1073/pnas.1113079108

Choi, G. B., Yim, Y. S., Wong, H., Kim, S., Kim, H., Kim, S. V., et al. (2016). The maternal interleukin-17a pathway in mice promotes autism-like phenotypes in offspring. Science 351, 933-939. doi: 10.1126/science.aad0314

Cieślinska, A., Kostyra, E., Chwała, B., Moszynska-Dumara, M., Fiedorowicz, E., Teodorowicz, M., et al. (2017). Vitamin D receptor gene polymorphisms associated with childhood autism. Brain Sci. 7:E115. doi: 10.3390/brainsci7090115

Clifford, S., Young, R., and Williamson, P. (2007). Assessing the early characteristics of autistic disorder using video analysis. J. Autism Dev. Disord. 37, 301-313. doi: 10.1007/s10803-006-0160-8

Coskun, M. A., Varghese, L., Reddoch, S., Castillo, E. M., Pearson, D. A., Loveland, K. A., et al. (2009). How somatic cortical maps differ in autistic and typical brains. Neuroreport 20, 175-179. doi: 10.1097/WNR.0b013e32831 f47d 1

Courchesne, E., Karns, C., Davis, H., Ziccardi, R., Carper, R., Tigue, Z., et al. (2011a). Unusual brain growth patterns in early life in patients with autistic disorder: An MRI study. Neurology 76, 2111-2111. doi: 10.1212/01.wnl.0000399191.79091.28

Courchesne, E., Mouton, P. R., Calhoun, M. E., Semendeferi, K., Ahrens-Barbeau, C., Hallet, M. J., et al. (2011b). Neuron number and size in prefrontal cortex of children with autism. JAMA 306, 2001-2010. doi: 10.1001/jama.2011.1638

Couture, S. M., Penn, D. L., Losh, M., Adolphs, R., Hurley, R., and Piven, J. (2010). Comparison of social cognitive functioning in schizophrenia and high functioning autism: more convergence than divergence. Psychol. Med. 40, 569-579. doi: 10.1017/S003329170999078X

Craig, F., Lamanna, A. L., Margari, F., Matera, E., Simone, M., and Margari, L. (2015). Overlap between autism spectrum disorders and attention deficit hyperactivity disorder: searching for distinctive/common clinical features. Autism Res. 8, 328-337. doi: 10.1002/aur.1449

Davis, M. C., Horan, W. P., Nurmi, E. L., Rizzo, S., Li, W., Sugar, C. A., et al. (2014). Associations between oxytocin receptor genotypes and social cognitive performance in individuals with schizophrenia. Schizophr. Res. 159, 353-357. doi: 10.1016/j.schres.2014.09.006

Dempster, E. L. (2007). Evidence of an association between the vasopressin V1b receptor gene (AVPR1B) and childhood-onset mood disorders. Arch. Gen. Psychiatry 64:1189. doi: 10.1001/archpsyc.64.10.1189

Dempster, E. L., Burcescu, I., Wigg, K., Kiss, E., Baji, I., Gadoros, J., et al. (2009). Further genetic evidence implicates the vasopressin system in childhood-onset mood disorders. Eur. J. Neurosci. 30, 1615-1619. doi: $10.1111 / \mathrm{j} .1460-9568.2009 .06930 . x$

Depino, A. M. (2017). Perinatal inflammation and adult psychopathology: From preclinical models to humans. Semin. Cell Dev. Biol. doi: 10.1016/j.semcdb.2017.09.010. [Epub ahead of print].

Deverman, B. E., and Patterson, P. H. (2009). Cytokines and CNS development. Neuron 64, 61-78. doi: 10.1016/j.neuron.2009.09.002

DiBenedictis, B. T., Nussbaum, E. R., Cheung, H. K., and Veenema, A. H. (2017). Quantitative mapping reveals age and sex differences in vasopressin, but not oxytocin, immunoreactivity in the rat social behavior neural network. J. Comp. Neurol. 525, 2549-2570. doi: 10.1002/cne.24216

Di Napoli, A., Warrier, V., Baron-Cohen, S., and Chakrabarti, B. (2014). Genetic variation in the oxytocin receptor (OXTR) gene is associated with asperger syndrome. Mol. Autism 5:48. doi: 10.1186/2040-2392-5-48

Ditzen, B., Schaer, M., Gabriel, B., Bodenmann, G., Ehlert, U., and Heinrichs, M. (2009). Intranasal oxytocin increases positive communication and reduces cortisol levels during couple conflict. Biol. Psychiatry 65, 728-731. doi: 10.1016/j.biopsych.2008.10.011

Domes, G., Heinrichs, M., Michel, A., Berger, C., and Herpertz, S. C. (2007). Oxytocin improves "mind-reading" in humans. Biol. Psychiatry 61, 731-733. doi: 10.1016/j.biopsych.2006.07.015

Donaldson, Z. R., and Young, L. J. (2008). Oxytocin, vasopressin, and the neurogenetics of sociality. Science 322, 900-904. doi: 10.1126/science.11 58668

Dumais, K. M., and Veenema, A. H. (2016). Vasopressin and oxytocin receptor systems in the brain: sex differences and sex-specific regulation of social behavior. Front. Neuroendocrinol. 40, 1-23. doi: 10.1016/j.yfrne.2015. 04.003 
Ebstein, R. P., Israel, S., Lerer, E., Uzefovsky, F., Shalev, I., Gritsenko, I., et al. (2009). Arginine vasopressin and oxytocin modulate human social behavior. Ann. N. Y. Acad. Sci. 1167, 87-102. doi: 10.1111/j.1749-6632.2009.04541.x

Ebstein, R. P., Knafo, A., Mankuta, D., Chew, S. H., and Lai, P. S. (2012). The contributions of oxytocin and vasopressin pathway genes to human behavior. Horm. Behav. 61, 359-379. doi: 10.1016/j.yhbeh.2011. 12.014

Ferguson, J. N., Young, L. J., Hearn, E. F., Matzuk, M. M., Insel, T. R., and Winslow, J. T. (2000). Social amnesia in mice lacking the oxytocin gene. Nat. Genet. 25, 284-288. doi: 10.1038/77040

Fombonne, E. (2002). Prevalence of childhood disintegrative disorder. Autism 6, 149-157. doi: $10.1177 / 1362361302006002002$

Fombonne, E. (2009). Epidemiology of pervasive developmental disorders. Pediatr. Res. 65, 591-598. doi: 10.1203/PDR.0b013e31819e7203

Francis, S. M., Kim, S. J., Kistner-Griffin, E., Guter, S., Cook, E. H., and Jacob, S. (2016). ASD and genetic associations with receptors for oxytocin and vasopressin-AVPR1A, AVPR1B, and OXTR. Front. Neurosci. 10:516. doi: $10.3389 /$ fnins. 2016.00516

Freeman, S. M., Inoue, K., Smith, A. L., Goodman, M. M., and Young, L. J. (2014). The neuroanatomical distribution of oxytocin receptor binding and mRNA in the male rhesus macaque (Macaca mulatta). Psychoneuroendocrinology 45, 128-141. doi: 10.1016/j.psyneuen.2014.03.023

Freitag, C. M. (2007). The genetics of autistic disorders and its clinical relevance: a review of the literature. Mol. Psychiatry 12, 2-22. doi: 10.1038/sj.mp.4001896

Fujiwara, T., Sanada, M., Kofuji, T., and Akagawa, K. (2016). Unusual social behavior in HPC-1/syntaxin1A knockout mice is caused by disruption of the oxytocinergic neural system. J. Neurochem. 138, 117-123. doi: $10.1111 /$ jnc. 13634

Fujiwara, T., Snada, M., Kofuji, T., Yoshikawa, T., and Akagawa, K. (2010). HPC-1/syntaxin 1A gene knockout mice show abnormal behavior possibly related to a disruption in 5-HTergic systems. Eur. J. Neurosci. 32, 99-107. doi: $10.1111 / j .1460-9568.2010 .07269 . x$

Furman, D. J., Chen, M. C., and Gotlib, I. H. (2011). Variant in oxytocin receptor gene is associated with amygdala volume. Psychoneuroendocrinology 36, 891-897. doi: 10.1016/j.psyneuen.2010.12.004

Gaugler, T., Klei, L., Sanders, S. J., Bodea, C. A., Goldberg, A. P., Lee, A. B., et al. (2014). Most genetic risk for autism resides with common variation. Nature Genetics 46, 881-885. doi: 10.1038/ng.3039

Geschwind, D. H. (2011). Genetics of autism spectrum disorders. Trends Cogn. Sci. 15, 409-416. doi: 10.1016/j.tics.2011.07.003

Gimpl, G., and Fahrenholz, F. (2001). The oxytocin receptor system: structure, function, and regulation. Physiol. Rev. 81, 629-683. doi: 10.1152/physrev.2001.81.2.629

Giulivi, C., Zhang, Y. F., Omanska-Klusek, A., Ross-Inta, C., Wong, S., HertzPicciotto, I., et al. (2010). Mitochondrial dysfunction in autism. JAMA 304, 2389-2396. doi: 10.1001/jama.2010.1706

Glessner, J. T., Li, J., Wang, D., March, M., Lima, L., Desai, A., et al. (2017). Copy number variation meta-analysis reveals a novel duplication at 9p24 associated with multiple neurodevelopmental disorders. Genome Med. 9:106. doi: 10.1186/s13073-017-0494-1

Glessner, J. T., Wang, K., Cai, G., Korvatska, O., Kim, C. E., Wood, S., et al. (2009). Autism genome-wide copy number variation reveals ubiquitin and neuronal genes. Nature 459, 569-573. doi: 10.1038/nature07953

Gregory, S. G., Connelly, J. J., Towers, A. J., Johnson, J., Biscocho, D., Markunas, C. A., et al. (2009). Genomic and epigenetic evidence for oxytocin receptor deficiency in autism. BMC Med. 7:62. doi: 10.1186/1741-701 5-7-62

Groen, W. B., Zwiers, M. P., van der Gaag, R. J., and Buitelaar, J. K. (2008). The phenotype and neural correlates of language in autism: an integrative review. Neurosci. Biobehav. Rev. 32, 1416-1425. doi: 10.1016/j.neubiorev.2008. 05.008

Guastella, A. J. and MacLeod, C. (2012). A critical review of the influence of oxytocin nasal spray on social cognition in humans: evidence and future directions. Horm. Behav. 61, 410-418. doi: 10.1016/j.yhbeh.2012. 01.002

Guo, H., Peng, Y., Hu, Z., Li, Y., Xun, G., Ou, J., et al. (2017). Genome-wide copy number variation analysis in a Chinese autism spectrum disorder cohort. Sci. Rep. 7:44155. doi: 10.1038/srep44155
Guoynes, C. D., Simmons, T. C., Downing, G. M., Jacob, S., Solomon, M., and Bales, K. L. (2017). Chronic intranasal oxytocin has dosedependent effects on central oxytocin and vasopressin systems in prairie voles (Microtus ochrogaster). Neuroscience 369, 292-302. doi: 10.1016/j.neuroscience.2017.11.037

Gupta, A. R., and State, M. W. (2007). Recent advances in the genetics of autism. Biol. Psychiatry 61, 429-437. doi: 10.1016/j.biopsych.2006.06.020

Halladay, A. K., Amaral, D., Aschner, M., Bolivar, V. J., Bowman, A., DiCicco-Bloom, E., et al. (2009). Animal models of autism spectrum disorders: information for neurotoxicologists. Neurotoxicology 30, 811-821. doi: 10.1016/j.neuro.2009.07.002

Hammock, E. A., and Young, L. J. (2002). Variation in the vasopressin V1a receptor promoter and expression: implications for inter- and intraspecific variation in social behaviour. Eur. J. Neurosci. 16, 399-402. doi: 10.1046/j.1460-9568.2002.02083.x

Hammock, E. A., and Young, L. J. (2006). Oxytocin, vasopressin and pair bonding: implications for autism. Philos. Trans. R. Soc. Lond. B Biol. Sci. 361, 2187-2198. doi: 10.1098/rstb.2006.1939

Haram, M., Tesli, M., Bettella, F., Djurovic, S., Andreassen, O. A., and Melle, I. (2015). Association between genetic variation in the oxytocin receptor gene and emotional withdrawal, but not between oxytocin pathway genes and diagnosis in psychotic disorders. Front. Hum. Neurosci. 9:9. doi: $10.3389 /$ fnhum.2015.00009

Harony, H., and Wagner, S. (2010). The contribution of oxytocin and vasopressin to mammalian social behavior: potential role in autism spectrum disorder. Neurosignals 18, 82-97. doi: 10.1159/000321035

Hazlett, H. C., Poe, M., Gerig, G., Smith, R. G., Provenzale, J., Ross, A., et al. (2005). Magnetic resonance imaging and head circumference study of brain size in autism: birth through age 2 years. Arch. Gen. Psychiatry 62, 1366-1376. doi: $10.1001 /$ archpsyc.62.12.1366

Heinrichs, M., and Domes, G. (2008). Neuropeptides and social behaviour: effects of oxytocin and vasopressin in humans. Prog. Brain Res. 170, 337-350. doi: 10.1016/S0079-6123(08)00428-7

Herman, J. P. (2012). Neural pathways of stress integration: relevance to alcohol abuse. Alcohol Res. 34, 441-447.

Hill, E. L., and Frith, U. (2003). Understanding autism: insights from mind and brain. Philos. Trans. R. Soc. Lond. Ser. B Biol. Sci. 358, 281-289. doi: 10.1098/rstb.2002.1209

Horvath, S., Xu, X., and Laird, N. M. (2001). The family based association test method: strategies for studying general genotype-phenotype associations. Eur. J. Hum. Genet. 9, 301-306. doi: 10.1038/sj.ejhg. 5200625

Hsiao, E. Y., McBride, S. W., Hsien, S., Sharon, G., Hyde, E. R., McCue, T., et al. (2013). Microbiota modulate behavioral and physiological abnormalities associated with neurodevelopmental disorders. Cell 155, 1451-1463. doi: 10.1016/j.cell.2013.11.024

Inglese, M. D., and Elder, J. H. (2009). Caring for children with autism spectrum disorder. Part I: prevalence, etiology, and core features. J. Pediatric Nurs. 24, 41-48. doi: 10.1016/j.pedn.2007.12.006

Inoue, H., Yamasue, H., Tochigi, M., Abe, O., Takei, K., Suga, M., et al. (2010). Association between the oxytocin receptor gene (OXTR) and amygdalar volume in healthy adults. Neurosci. Res. 68:e205. doi: 10.1016/j.neures.2010.07.2479

Insel, T. R., O’Brien, D. J., and Leckman, J. F. (1999). Oxytocin, vasopressin, and autism: is there a connection? Biol. Psychiatry, 45, 145-157.

Insel, T. R., and Shapiro, L. E. (1992). Oxytocin receptor distribution reflects social organization in monogamous and polygamous voles. Proc. Natl. Acad. Sci. U.S.A. 89, 5981-5985. doi: 10.1073/pnas.89.13.5981

Insel, T. R., Wang, Z. X., and Ferris, C. F. (1994). Patterns of brain vasopressin receptor distribution associated with social organization in microtine rodents. J. Neurosci. 14, 5381-5392.

Ishizuka, K., Fujita, Y., Kawabata, T., Kimura, H., Iwayama, Y., Inada, T., et al. (2017). Rare genetic variants in CX3CR1 and their contribution to the increased risk of schizophrenia and autism spectrum disorders. Transl. Psychiatry 7:e1184. doi: 10.1038/tp.2017.173

Israel, S., Lerer, E., Shalev, I., Uzefovsky, F., Reibold, M., Bachner-Melman, R., et al. (2008). Molecular genetic studies of the arginine vasopressin 1a receptor (AVPR1a) and the oxytocin receptor (OXTR) in human behaviour: from 
autism to altruism with some notes in between. Prog. Brain Res. 170, 435-449. doi: 10.1016/S0079-6123(08)00434-2

Israel, S., Lerer, E., Shalev, I., Uzefovsky, F., Riebold, M., Laiba, E., et al. (2009). The oxytocin receptor (OXTR) contributes to prosocial fund allocations in the dictator game and the social value orientations task. PLoS ONE 4:e5535. doi: 10.1371/journal.pone.0005535

Jack, A., Connelly, J. J., and Morris, J. P. (2012). DNA methylation of the oxytocin receptor gene predicts neural response to ambiguous social stimuli. Front. Hum. Neurosci. 6:280. doi: 10.3389/fnhum.2012.00280

Jacob, S., Brune, C. W., Carter, C. S., Leventhal, B. L., Lord, C., and Cook, E. H., et al. (2007). Association of the oxytocin receptor gene (OXTR) in Caucasian children and adolescents with autism. Neurosci. Lett. 417, 6-9. doi: 10.1016/j.neulet.2007.02.001

Johansson, A., Bergman, H., Corander, J., Waldman, I. D., Karrani, N., Salo, B., et al. (2012). Alcohol and aggressive behavior in men-moderating effects of oxytocin receptor gene (OXTR) polymorphisms. Genes Brain Behav. 11, 214-221. doi: 10.1111/j.1601-183X.2011.00744.x

Johnson, R. T. (1994). Infections during pregnancy. Adv. Neurol. 64, 153-162.

Kanner, L. (1968). Autistic disturbances of affective contact. Acta Paedopsychiatr. $35,100-136$

Kantojärvi, K., Oikkonen, J., Kotala, I., Kallela, J., Vanhala, R., Onkamo, P., et al. (2015). Association and promoter analysis of AVPR1A in finnish autism families. Autism Res. 8, 634-639. doi: 10.1002/aur.1473

Keck, M. E., Kern, N., Erhardt, A., Unschuld, P. G., Ising, M., Salyakina, D., et al. (2008). Combined effects of exonic polymorphisms in CRHR1 and AVPR1B genes in a case/control study for panic disorder. Am. J. Med. Genet. Part B Neuropsychiatr. Genet. 147B, 1196-1204. doi: 10.1002/ajmg.b. 30750

Kim, S. J., Young, L. J., Gonen, D., Veenstra-VanderWeele, J., Courchesne, R., Courchesne, E., et al. (2002). Transmission disequilibrium testing of arginine vasopressin receptor 1A (AVPR1A) polymorphisms in autism. Mol. Psychiatry 7, 503-507. doi: 10.1038/sj.mp.4001125

Knafo, A., Israel, S., Darvasi, A., Bachner-Melman, R., Uzefovsky, F., Cohen, L., et al. (2008). Individual differences in allocation of funds in the dictator game associated with length of the arginine vasopressin 1a receptor RS3 promoter region and correlation between RS3 length and hippocampal mRNA. Genes Brain Behav. 7, 266-275. doi: 10.1111/j.1601-183X.2007.00341.x

Knapp, M., Romeo, R., and Beecham, J. (2009). Economic cost of autism in the UK. Autism 13, 317-336. doi: 10.1177/1362361309104246

Kogan, A., Saslow, L. R., Impett, E. A., Oveis, C., Keltner, D., and Rodrigues Saturn, S. (2011). Thin-slicing study of the oxytocin receptor (OXTR) gene and the evaluation and expression of the prosocial disposition. Proc. Natl. Acad. Sci. U.S.A. 108, 19189-19192. doi: 10.1073/pnas.1112658108

Kosfeld, M., Heinrichs, M., Zak, P. J., Fischbacher, U., and Fehr, E. (2005). Oxytocin increases trust in humans. Nature 435, 673-676. doi: 10.1038/nature03701

Kranz, T. M., Kopp, M., Waltes, R., Sachse, M., Duketis, E., Jarczok, T. A., et al. (2016). Meta-analysis and association of two common polymorphisms of the human oxytocin receptor gene in autism spectrum disorder. Autism Res. 9, 1036-1045. doi: 10.1002/aur.1597

Ku, K. M., Weir, R. K., Silverman, J. L., Berman, R. F., and Bauman, M. D. (2016). Behavioral phenotyping of juvenile long-evans and sprague-dawley rats: implications for preclinical models of autism spectrum disorders. PLoS ONE 11:e0158150. doi: 10.1371/journal.pone.0158150

Kulage, K. M., Smaldone, A. M., and Cohn, E. G. (2014). How will DSM-5 affect autism diagnosis? A systematic literature review and meta-analysis. J. Autism Dev. Disord. 44, 1918-1932. doi: 10.1007/s10803-014-2065-2

Kumar, R. A., and Christian, S. L. (2009). Genetics of autism spectrum disorders. Curr. Neurol. Neurosci. Rep. 9, 188-197. doi: 10.1007/s11910-009-0029-2

Kwakye, L. D., Foss-Feig, J. H., Cascio, C. J., Stone, W. L., and Wallace, M. T. (2011). Altered auditory and multisensory temporal processing in autism spectrum disorders. Front. Integr. Neurosci. 4:129. doi: 10.3389/fnint.2010.00129

Lee, B. K., Magnusson, C., Gardner, R. M., Blomström, Å., Newschaffer, C. J., Burstyn, I., et al. (2015). Maternal hospitalization with infection during pregnancy and risk of autism spectrum disorders. Brain Behav. Immun. 44, 100-105. doi: 10.1016/j.bbi.2014.09.001

Lerer, E., Levi, S., Salomon, S., Darvasi, A., Yirmiya, N., and Ebstein, R. P. (2008). Association between the oxytocin receptor (OXTR) gene and autism: relationship to vineland adaptive behavior scales and cognition. Mol. Psychiatry 13, 980-988. doi: 10.1038/sj.mp.4002087

Lesse, A., Rether, K., Gröger, N., Braun, K., and Bock, J. (2017). Chronic postnatal stress induces depressive-like behavior in male mice and programs second-hit stress-induced gene expression patterns of OxtR and AvpRla in adulthood. Mol. Neurobiol. 54, 4813-4819. doi: 10.1007/s12035-016-0 043-8

Levin, R., Heresco-Levy, U., Bachner-Melman, R., Israel, S., Shalev, I., and Ebstein, R. P. (2009). Association between arginine vasopressin 1a receptor (AVPR1a) promoter region polymorphisms and prepulse inhibition. Psychoneuroendocrinology 34, 901-908. doi: 10.1016/j.psyneuen.2008.12.014

Lim, M. M., Bielsky, I. F., and Young, L. J. (2005). Neuropeptides and the social brain: potential rodent models of autism. Int. J. Dev. Neurosci. 23, 235-243. doi: 10.1016/j.ijdevneu.2004.05.006

Lintas, C., and Persico, A. M. (2009). Autistic phenotypes and genetic testing: state-of-the-art for the clinical geneticist. J. Med. Genet. 46, 1-8. doi: 10.1136/jmg.2008.060871

Li, Q., Han, Y., Dy, A. B. C., and Hagerman, R. J. (2017). The gut microbiota and autism spectrum disorders. Front. Cell. Neurosci. 11:120. doi: 10.3389/fncel.2017.00120

Liu, X., Kawamura, Y., Shimada, T., Otowa, T., Koishi, S., Sugiyama, T., et al. (2010). Association of the oxytocin receptor (OXTR) gene polymorphisms with autism spectrum disorder (ASD) in the Japanese population. J. Hum. Genet. 55, 137-141. doi: 10.1038/jhg.2009.140

LoParo, D., and Waldman, I. D. (2015). The oxytocin receptor gene (OXTR) is associated with autism spectrum disorder: a meta-analysis. Mol. Psychiatry 20, 640-646. doi: 10.1038/mp.2014.77

Lord, C., Risi, S., Lambrecht, L., Cook, E. H. Jr., Leventhal, B. L., DiLavore, P. C., et al. (2000). The autism diagnostic observation schedule-generic: a standard measure of social and communication deficits associated with the spectrum of autism. J. Autism Dev. Disord. 30, 205-223. doi: 10.1023/A:10055924 01947

Lucht, M. J., Barnow, S., Sonnenfeld, C., Rosenberger, A., Grabe, H. J., Schroeder, W., et al. (2009). Associations between the oxytocin receptor gene (OXTR) and affect, loneliness and intelligence in normal subjects. Prog. Neuropsychopharmacol. Biol. Psychiatry 33, 860-866. doi: 10.1016/j.pnpbp.2009.04.004

Luppino, D., Moul, C., Hawes, D. J., Brennan, J., and Dadds, M. R. (2014). Association between a polymorphism of the vasopressin 1B receptor gene and aggression in children. Psychiatr. Genet. 24, 185-190. doi: 10.1097/YPG.0000000000000036

Malik, A. I., Zai, C. C., Abu, Z., Nowrouzi, B., and Beitchman, J. H. (2012). The role of oxytocin and oxytocin receptor gene variants in childhood-onset aggression. Genes Brain Behav. 11, 545-551. doi: 10.1111/j.1601-183X.2012. 00776.x

Malkova, N. V., Yu, C. Z., Hsiao, E. Y., Moore, M. J., and Patterson, P. H. (2012). Maternal immune activation yields offspring displaying mouse versions of the three core symptoms of autism. Brain Behav. Immun. 26, 607-616. doi: 10.1016/j.bbi.2012.01.011

Manning, M., Stoev, S., Chini, B., Durroux, T., Mouillac, B., and Guillon, G. (2008). Peptide and non-peptide agonists and antagonists for the vasopressin and oxytocin V1a, V1b, V2 and OT receptors: research tools and potential therapeutic agents. Prog. Brain Res. 170, 473-512. doi: 10.1016/S0079-6123(08)00437-8

Marshall, C. R., Noor, A., Vincent, J. B., Lionel, A. C., Feuk, L., Skaug, J., et al. (2008). Structural variation of chromosomes in autism spectrum disorder. Am. J. Hum. Genet. 82, 477-488. doi: 10.1016/j.ajhg.2007.12.009

Marusak, H. A., Furman, D. J., Kuruvadi, N., Shattuck, D. W., Joshi, S. H., Joshi, A. A., et al. (2015). Amygdala responses to salient social cues vary with oxytocin receptor genotype in youth. Neuropsychologia 79(Pt A), 1-9. doi: 10.1016/j.neuropsychologia.2015.10.015

Mazina, V., Gerdts, J., Trinh, S., Ankenman, K., Ward, T., Dennis, M. Y., et al. (2015). Epigenetics of autism-related impairment: copy number variation and maternal infection. J. Dev. Behav. Pediatr. 36, 61-67. doi: 10.1097/DBP.0000000000000126

McDonald, N. M., Baker, J. K., and Messinger, D. S. (2016). Oxytocin and parentchild interaction in the development of empathy among children at risk for autism. Dev. Psychol. 52, 735-745. doi: 10.1037/dev0000104 
Medzhitov, R. (2001). Toll-like receptors and innate immunity. Nat. Rev. Immunol. 1, 135-145. doi: $10.1038 / 35100529$

Meyer, U., Feldon, J., and Dammann, O. (2011). Schizophrenia and autism: both shared and disorder-specific pathogenesis via perinatal inflammation? Pediatr. Res. 69(5 Pt 2), 26R-33R. doi: 10.1203/PDR.0b013e318212c196

Meyer, U., Feldon, J., and Fatemi, S. H. (2009). In-vivo rodent models for the experimental investigation of prenatal immune activation effects in neurodevelopmental brain disorders. Neurosci. Biobehav. Rev. 33, 1061-1079. doi: 10.1016/j.neubiorev.2009.05.001

Meyer-Lindenberg, A., Kolachana, B., Gold, B., Olsh, A., Nicodemus, K. K., Mattay, V., et al. (2009). Genetic variants in AVPR1A linked to autism predict amygdala activation and personality traits in healthy humans. Mol. Psychiatry 14, 968-975. doi: $10.1038 / \mathrm{mp} .2008 .54$

Meyer-Lindenberg, A., and Tost, H. (2012). Neural mechanisms of social risk for psychiatric disorders. Nat. Neurosci. 15, 663-668. doi: 10.1038/ nn. 3083

Miller, M., Bales, K. L., Taylor, S. L., Yoon, J., Hostetler, C. M., Carter, C. S., et al. (2013). Oxytocin and vasopressin in children and adolescents with autism spectrum disorders: sex differences and associations with symptoms. Autism Res. 6, 91-102. doi: 10.1002/aur.1270

Miller, V. M., Zhu, Y., Bucher, C., McGinnis, W., Ryan, L. K., Siegel, A., et al. (2013). Gestational flu exposure induces changes in neurochemicals, affiliative hormones and brainstem inflammation, in addition to autism-like behaviors in mice. Brain Behav. Immun. 33, 153-163. doi: 10.1016/j.bbi.2013. 07.002

Modi, M. E., and Young, L. J. (2012). The oxytocin system in drug discovery for autism: animal models and novel therapeutic strategies. Horm. Behav. 61, 340-350. doi: 10.1016/j.yhbeh.2011.12.010

Montag, C., Sindermann, C., Melchers, M., Jung, S., Luo, R., Becker, B., et al. (2017). A functional polymorphism of the OXTR gene is associated with autistic traits in Caucasian and Asian populations. Am. J. Med. Genet. Part B Neuropsychiatr. Genet. 174, 808-816. doi: 10.1002/ajmg.b. 32596

Moons, W. G., Way, B. M., and Taylor, S. E. (2014). Oxytocin and vasopressin receptor polymorphisms interact with circulating neuropeptides to predict human emotional reactions to stress. Emotion 14, 562-572. doi: $10.1037 / \mathrm{a} 0035503$

Moore, S. J., Turnpenny, P., Quinn, A., Glover, S., Lloyd, D. J., Montgomery, T., et al. (2000). A clinical study of 57 children with fetal anticonvulsant syndromes. J. Med. Genet. 37, 489-497. doi: 10.1136/jmg.37.7.489

Mosconi, M. W., Cody-Hazlett, H., Poe, M. D., Gerig, G., Gimpel-Smith, R., and Piven, J. (2009). Longitudinal study of amygdala volume and joint attention in 2- to 4-year-old children with autism. Arch. Gen. Psychiatry 66, 509-516. doi: 10.1001/archgenpsychiatry.2009.19

Murakami, G., Hunter, R. G., Fontaine, C., Ribeiro, A., and Pfaff, D. (2011). Relationships among estrogen receptor, oxytocin and vasopressin gene expression and social interaction in male mice. Eur. J. Neurosci. 34, 469-477. doi: $10.1111 / j .1460-9568.2011 .07761 . x$

Muráni, E., Ponsuksili, S., D’Eath, R. B., Turner, S. P., Kurt, E., Evans, G., et al. (2010). Association of HPA axis-related genetic variation with stress reactivity and aggressive behaviour in pigs. BMC Genet. 11:74. doi: 10.1186/1471-2156-11-74

Nemirovsky, S. I., Córdoba, M., Zaiat, J. J., Completa, S. P., Vega, P. A., González-Morón, D., et al. (2015). Whole genome sequencing reveals a de novo SHANK3 mutation in familial autism spectrum disorder. PLOS ONE 10:e0116358. doi: 10.1371/journal.pone.0116358

Neumann, I. D. (2008). Brain oxytocin: a key regulator of emotional and social behaviours in both females and males. J. Neuroendocrinol. 20, 858-865. doi: 10.1111/j.1365-2826.2008.01726.x

Nicolini, C., and Fahnestock, M. (2017). The valproic acid-induced rodent model of autism. Exp. Neurol. 299(Pt A), 217-227. doi: 10.1016/j.expneurol.2017.04.017

Nithianantharajah, J., Balasuriya, G. K., Franks, A. E., and Hill-Yardin, E. L. (2017). Using animal models to study the role of the gut-brain axis in autism. Curr. Dev. Dis. Rep. 4, 28-36. doi: 10.1007/s40474-017-0111-4

Nyffeler, J., Walitza, S., Bobrowski, E., Gundelfinger, R., and Grünblatt, E. (2014). Association study in siblings and case-controls of serotonin- and oxytocin-related genes with high functioning autism. J. Mol. Psychiatry 2:1. doi: 10.1186/2049-9256-2-1

Pagani, J. H., Zhao, M., Cui, Z., Avram, S. K., Caruana, D. A., Dudek, S. M., et al. (2015). Role of the vasopressin $1 \mathrm{~b}$ receptor in rodent aggressive behavior and synaptic plasticity in hippocampal area CA2. Mol. Psychiatry 20, 490-499. doi: $10.1038 / \mathrm{mp} .2014 .47$

Panagiotidi, M., Overton, P. G., and Stafford, T. (2017). Co-occurrence of ASD and ADHD traits in an adult population. J. Atten. Disord. doi: 10.1177/1087054717720720. [Epub ahead of print].

Panksepp, J. (1992). Oxytocin effects on emotional processes: separation distress, social bonding, and relationships to psychiatric disorders. Ann. N. Y. Acad. Sci. 652, 243-252. doi: 10.1111/j.1749-6632.1992.tb34359.x

Paré, P., Paixão-Côrtes, V. R., Tovo-Rodrigues, L., Vargas-Pinilla, P., Viscardi, L. H., Salzano, F. M., et al. (2016). Oxytocin and arginine vasopressin receptor evolution: implications for adaptive novelties in placental mammals. Genet. Mol. Biol. 39, 646-657. doi: 10.1590/1678-4685-gmb-2015-0323

Parker, K. J., Garner, J. P., Libove, R. A., Hyde, S. A., Hornbeak, K. B., Carson, D. S., et al. (2014). Plasma oxytocin concentrations and OXTR polymorphisms predict social impairments in children with and without autism spectrum disorder. Proc. Natl. Acad. Sci. U.S.A. 111, 12258-12263. doi: 10.1073/pnas.1402236111

Patterson, P. H. (2011). Maternal infection and immune involvement in autism. Trends Mol. Med. 17, 389-394. doi: 10.1016/j.molmed.2011.03.001

Peñagarikano, O. (2017). Oxytocin in animal models of autism spectrum disorder. Dev. Neurobiol. 77, 202-213. doi: 10.1007/7854_2017_15

Peñagarikano, O., Lazaro, M. T., Lu, X. H., Gordon, A., Dong, H., Lam, H. A., et al. (2015). Exogenous and evoked oxytocin restores social behavior in the Cntnap2 mouse model of autism. Sci. Transl. Med. 7:271ra8. doi: 10.1126/scitranslmed.3010257

Platt, M. L., Seyfarth, R. M., and Cheney, D. L. (2016). Adaptations for social cognition in the primate brain. Philos. Trans. R. Soc. Lond. Ser. B Biol. Sci. 371:20150096. doi: 10.1098/rstb.2015.0096

Poulin, M. J., Holman, E. A., and Buffone, A. (2012). The neurogenetics of nice: receptor genes for oxytocin and vasopressin interact with threat to predict prosocial behavior. Psychol. Sci. 23, 446-452. doi: 10.1177/0956797611428471

Prata, J., Santos, S. G., Almeida, M. I., Coelho, R., and Barbosa, M. A. (2017). Bridging autism spectrum disorders and schizophrenia through inflammation and biomarkers - pre-clinical and clinical investigations. J. Neuroinflammation 14:179. doi: 10.1186/s12974-017-0938-y

Procyshyn, T. L., Hurd, P. L., and Crespi, B. J. (2016). Association testing of vasopressin receptor 1a microsatellite polymorphisms in non-clinical autism spectrum phenotypes. Autism Res. 10, 750-756. doi: 10.1002/aur.1716

Rasalam, A. D., Hailey, H., Williams, J. H., Moore, S. J., Turnpenny, P. D., Lloyd, D. J., et al. (2005). Characteristics of fetal anticonvulsant syndrome associated autistic disorder. Dev. Med. Child Neurol. 47, 551-555. doi: $10.1017 /$ S0012162205001076

Redcay, E., and Courchesne, E. (2005). When is the brain enlarged in autism? A meta-analysis of all brain size reports. Biol. Psychiatry 58, 1-9. doi: 10.1016/j.biopsych.2005.03.026

Rijlaarsdam, J., van IJzendoorn, M. H., Verhulst, F. C., Jaddoe, V. W. V., Felix, J. F., Tiemeier, H., et al. (2017). Prenatal stress exposure, oxytocin receptor gene (OXTR) methylation, and child autistic traits: the moderating role of OXTR rs53576 genotype. Autism Res. 10, 430-438. doi: 10.1002/aur.1681

Rodrigues, S. M., Saslow, L. R., Garcia, N., John, O. P., and Keltner, D. (2009). Oxytocin receptor genetic variation relates to empathy and stress reactivity in humans. Proc. Natl. Acad. Sci. U.S.A. 106, 21437-21441. doi: 10.1073/pnas.0909579106

Roper, J., O'Carroll, A. M., Young, W. III., and Lolait, S. (2011). The vasopressin Avprlb receptor: molecular and pharmacological studies. Stress. 14, 98-115. doi: $10.3109 / 10253890.2010 .512376$

Rossignol, D. A., and Frye, R. E. (2012). A review of research trends in physiological abnormalities in autism spectrum disorders: immune dysregulation, inflammation, oxidative stress, mitochondrial dysfunction and environmental toxicant exposures. Mol. Psychiatry 17, 389-401. doi: 10.1038/mp.2011.165

Rutter, M. (2005). Autism research: lessons from the past and prospects for the future. J. Autism Dev. Disord. 35, 241-257. doi: 10.1007/s10803-0042003-9 
Sala, M., Braida, D., Lentini, D., Busnelli, M., Bulgheroni, E., Capurro, V., et al. (2011). Pharmacologic rescue of impaired cognitive flexibility, social deficits, increased aggression, and seizure susceptibility in oxytocin receptor null mice: a neurobehavioral model of autism. Biol. Psychiatry 69, 875-882. doi: 10.1016/j.biopsych.2010.12.022

Sanders, S. J., He, X., Willsey, A. J., Ercan-Sencicek, A. G., Samocha, K. E., Cicek, A. E., et al. (2015). Insights into autism spectrum disorder genomic architecture and biology from 71 risk loci. Neuron 87, 1215-1233. doi: 10.1016/j.neuron.2015.09.016

Sandin, S., Lichtenstein, P., Kuja-Halkola, R., Larsson, H., Hultman, C. M., and Reichenberg, A. (2014). The familial risk of autism. JAMA 311, 1770-1777. doi: 10.1001/jama.2014.4144

Saphire-Bernstein, S., Way, B. M., Kim, H. S., Sherman, D. K., and Taylor, S. E. (2011). Oxytocin receptor gene (OXTR) is related to psychological resources. Proc. Natl. Acad. Sci. U.S.A. 108, 15118-15122. doi: 10.1073/pnas.1113137108

Schneider-Hassloff, H., Straube, B., Jansen, A., Nuscheler, B., Wemken, G., Witt, S. H., et al. (2016). Oxytocin receptor polymorphism and childhood social experiences shape adult personality, brain structure and neural correlates of mentalizing. Neuroimage 134, 671-684. doi: 10.1016/j.neuroimage.2016.04.009

Schumann, C. M., Bloss, C. S., Barnes, C. C., Wideman, G. M., Carper, R. A., Akshoomoff, N., et al. (2010). Longitudinal magnetic resonance imaging study of cortical development through early childhood in autism. J. Neurosci. 30, 4419-4427. doi: 10.1523/JNEUROSCI.5714-09.2010

Schwartzer, J. J., Onore, C. E., Rose, D., and Ashwood, P. (2017). C57BL/6J bone marrow transplant increases sociability in BTBR T Itpr3tf/J mice. Brain Behav. Immun. 59, 55-61. doi: 10.1016/j.bbi.2016.05.019

Schwartzer, J. J., Careaga, M., Onore, C. E., Rushakoff, J. A., Berman, R. F., and Ashwood, P. (2013). Maternal immune activation and strain specific interactions in the development of autism-like behaviors in mice. Transl. Psychiatry 3:e240. doi: 10.1038/tp.2013.16

Sebat, J., Lakshmi, B., Malhotra, D., Troge, J., Lese-Martin, C., Walsh, T., et al. (2007). Strong association of de novo copy number mutations with autism. Science 316, 445-449. doi: 10.1126/science.1138659

Shou, X. J., Xu, X. J., Zeng, X. Z., Liu, Y., Yuan, H. S., Xing, Y., et al. (2017). A volumetric and functional connectivity MRI study of brain arginine-vasopressin pathways in autistic children. Neurosci. Bull. 33, 130-142. doi: 10.1007/s12264-017-0109-2

Simonoff, E., Pickles, A., Charman, T., Chandler, S., Loucas, T., and Baird, G. (2008). Psychiatric disorders in children with autism spectrum disorders: prevalence, comorbidity, and associated factors in a populationderived sample. J. Am. Acad. Child Adolesc. Psychiatry 47, 921-929. doi: 10.1097/CHI.0b013e318179964f

Skylark, W. J., and Baron-Cohen, S. (2017). Initial evidence that nonclinical autistic traits are associated with lower income. Mol. Autism 8:61. doi: 10.1186/s13229-017-0179-Z

Smith, I. C., Reichow, B., and Volkmar, F. R. (2015). The Effects of DSM5 criteria on number of individuals diagnosed with autism spectrum disorder: a systematic review. J. Autism Dev. Disord. 45, 2541-2552. doi: $10.1007 /$ s10803-015-2423-8

Snow, A. V., Lecavalier, L., and Houts, C. (2009). The structure of the autism diagnostic interview-revised: diagnostic and phenotypic implications. J. Child Psychol. Psychiatry 50, 734-742. doi: 10.1111/j.1469-7610.2008.02018.x

Stewart, A. M., and Kalueff, A. V. (2015). Developing better and more valid animal models of brain disorders. Behav. Brain Res. 276, 28-31. doi: 10.1016/j.bbr.2013.12.024

Steyaert, J. G., and De la Marche, W. (2008). What's new in autism? Eur. J. Pediatr. 167, 1091-1101. doi: 10.1007/s00431-008-0764-4

Stoop, R. (2012). Neuromodulation by oxytocin and vasopressin. Neuron 76, 142-159. doi: 10.1016/j.neuron.2012.09.025

Strasser, L., Downes, M., Kung, J., Cross, J. H., and De Haan, M. (2017). Prevalence and risk factors for autism spectrum disorder in epilepsy: a systematic review and meta-analysis. Dev. Med. Child Neurol. 60, 19-29. doi: 10.1111/dmcn.13598

Sturmey, P., and Dalfern, S. (2014). The effects of DSM5 autism diagnostic criteria on number of individuals diagnosed with autism spectrum disorders: a systematic review. Rev. J. Autism Dev. Dis. 1, 249-252. doi: 10.1007/s40489-014-0016-7
St-Hilaire, S., Ezike, V. O., Stryhn, H., and Thomas, M. A. (2012). An ecological study on childhood autism. Int. J. Health Geogr. 11:44. doi: 10.1186/1476-072X-11-44

Suzuki, T., Miyaki, K., Eguchi, H., and Tsutsumi, A. (2017). Distribution of autistic traits and their association with sociodemographic characteristics in Japanese workers. Autism. doi: 10.1177/1362361317716605. [Epub ahead of print].

Südhof, T. C., and Rothman, J. E. (2009). Membrane fusion: grappling with SNARE and SM proteins. Science 323, 474-477. doi: 10.1126/science.1161748

Szatmari, P., Georgiades, S., Bryson, S., Zwaigenbaum, L., Roberts, W., Mahoney, W., et al. (2006). Investigating the structure of the restricted, repetitive behaviours and interests domain of autism. J. Child Psychol. Psychiatry 47, 582-590. doi: 10.1111/j.1469-7610.2005.01537.x

Tansey, K. E., Brookes, K. J., Hill, M. J., Cochrane, L. E., Gill, M., Skuse, D., et al. (2010). Oxytocin receptor (OXTR) does not play a major role in the aetiology of autism: genetic and molecular studies. Neurosci. Lett. 474, 163-167. doi: 10.1016/j.neulet.2010.03.035

Tansey, K. E., Hill, M. J., Cochrane, L. E., Gill, M., Anney, R. J., and Gallagher, L. (2011). Functionality of promoter microsatellites of arginine vasopressin receptor 1A (AVPR1A): implications for autism. Mol. Autism 2:3. doi: 10.1186/2040-2392-2-3

Taylor, S. E., Saphire-Bernstein, S., and Seeman, T. E. (2010). Are plasma oxytocin in women and plasma vasopressin in men biomarkers of distressed pair-bond relationships? Psychol. Sci. 21, 3-7. doi: 10.1177/09567976093 56507

The Autism Spectrum Disorders Working Group of The Psychiatric Genomics Consortium (2017). Meta-analysis of GWAS of over 16,000 individuals with autism spectrum disorder highlights a novel locus at 10q24.32 and a significant overlap with schizophrenia. Mol. Autism 8:21. doi: 10.1186/s13229-017-0 $137-9$

Thibonnier, M. (2004). Genetics of vasopressin receptors. Curr. Hypertens. Rep. 6, 21-26. doi: 10.1007/s11906-004-0006-8

Thibonnier, M., Coles, P., Thibonnier, A., and Shoham, M. (2002). Molecular pharmacology and modeling of vasopressin receptors. Prog. Brain Res. 139, 179-196. doi: 10.1016/S0079-6123(02)39016-2

Thibonnier, M., Graves, M. K., Wagner, M. S., Chatelain, N., Soubrier, F., Corvol, P., et al. (2000). Study of V1-vascular vasopressin receptor gene microsatellite polymorphisms in human essential hypertension. J. Mol. Cell. Cardiol. 32, 557-564. doi: 10.1006/jmcc.2000.1108

Thompson, M. R., Callaghan, P. D., Hunt, G. E., Cornish, J. L., and McGregor, I. S. (2007). A role for oxytocin and 5-HT(1A) receptors in the prosocial effects of 3,4 methylenedioxymethamphetamine ("ecstasy"). Neuroscience 146, 509-514. doi: 10.1016/j.neuroscience.2007.02.032

Thompson, R. J., Parker, K. J., Hallmayer, J. F., Waugh, C. E., and Gotlib, I. H. (2011). Oxytocin receptor gene polymorphism (rs2254298) interacts with familial risk for psychopathology to predict symptoms of depression and anxiety in adolescent girls. Psychoneuroendocrinology 36, 144-147. doi: 10.1016/j.psyneuen.2010.07.003

Tick, B., Bolton, P., Happé, F., Rutter, M., and Rijsdijk, F. (2016). Heritability of autism spectrum disorders: a meta-analysis of twin studies. J. Child. Psychol. Psychiatry. 57, 585-595. doi: 10.1111/jcpp.12499

Tickerhoof, M. C., and Smith, A. S. (2017). Vasopressinergic neurocircuitry regulating social attachment in a Monogamous species. Front. Endocrinol. 8:265. doi: 10.3389/fendo.2017.00265

Tommerdahl, M., Tannan, V., Cascio, C. J., Baranek, G. T., and Whitsel, B. L. (2007). Vibrotactile adaptation fails to enhance spatial localization in adults with autism. Brain Res. 1154, 116-123. doi: 10.1016/j.brainres.2007. 04.032

Tordjman, S., Somogyi, E., Coulon, N., Kermarrec, S., Cohen, D., Bronsard, G., et al. (2014). Gene $\mathrm{x}$ environment interactions in autism spectrum disorders: role of epigenetic mechanisms. Front. Psychiatry. 5:53. doi: 10.3389/fpsyt.2014.00053

Tost, H., Kolachana, B., Hakimi, S., Lemaitre, H., Verchinski, B. A., Mattay, V. S., et al. (2010). A common allele in the oxytocin receptor gene (OXTR) impacts prosocial temperament and human hypothalamic-limbic structure and function. Proc. Natl. Acad. Sci. U.S.A. 107, 13936-13941. doi: 10.1073/pnas.1003296107 
Tseng, P. T., Chen, Y. W., Stubbs, B., Carvalho, A. F., Whiteley, P., Tang, C. H., et al. (2017). Maternal breastfeeding and autism spectrum disorder in children: A systematic review and meta-analysis. Nutr. Neurosci. doi: 10.1080/1028415X. 2017.1388598. [Epub ahead of print].

Uzefovsky, F., Shalev, I., Israel, S., Edelman, S., Raz, Y., Mankuta, D., et al. (2015). Oxytocin receptor and vasopressin receptor 1a genes are respectively associated with emotional and cognitive empathy. Horm. Behav. 67, 60-65. doi: 10.1016/j.yhbeh.2014.11.007

Vanderschuren, L. J., and Trezza, V. (2013). What the laboratory rat has taught us about social play behavior: role in behavioral development and neural mechanisms. Curr. Top. Behav. Neurosci. 16, 189-212. doi: 10.1007/978-3-662-45758-0_268

van West, D., Del-Favero, J., Deboutte, D., Van Broeckhoven, C., and Claes, S. (2009). Arginine vasopressin receptor gene-based single-nucleotide polymorphism analysis in attention deficit hyperactivity disorder. Psychiatr. Genet. 19, 102-103. doi: 10.1097/YPG.0b013e32832a0b2b

Veenema, A. H., Beiderbeck, D. I., Lukas, M., and Neumann, I. D. (2010). Distinct correlations of vasopressin release within the lateral septum and the bed nucleus of the stria terminalis with the display of intermale aggression. Horm. Behav. 58, 273-281. doi: 10.1016/j.yhbeh.2010.03.006

Veenema, A. H., and Neumann, I. D. (2008). Central vasopressin and oxytocin release: regulation of complex social behaviours. Prog. Brain Res. 170, 261-276. doi: 10.1016/S0079-6123(08)00422-6

Veenstra-VanderWeele, J., and Cook, E. H. (2004). Molecular genetics of autism spectrum disorder. Mol. Psychiatry 9, 819-832. doi: 10.1038/sj.mp.40 01505

Verhallen, R. J., Bosten, J. M., Goodbourn, P. T., Lawrance-Owen, A. J., Bargary, G., and Mollon, J. D. (2017). The oxytocin receptor gene ( OXTR) and face recognition. Psychol. Sci. 28, 47-55. doi: 10.1177/0956797616672269

Volk, H. E., Lurmann, F., Penfold, B., Hertz-Picciotto, I., and McConnell, R. (2013). Traffic-related air pollution, particulate matter, and autism. JAMA 70:71. doi: 10.1001/jamapsychiatry.2013.266

Volkmar, F., Cook, E. Jr., Pomeroy, J., Realmuto, G., and Tanguay, P. (1999). Summary of the practice parameters for the assessment and treatment of children, adolescents, and adults with autism and other pervasive developmental disorders. Am. Acad. Child Adolesc. Psychiatry 38, 1611-1616. doi: 10.1016/S0890-8567(99)80003-3

Vorstman, J. A., Staal, W. G., van Daalen, E., van Engeland, H., Hochstenbach, P. F., and Franke, L. (2006). Identification of novel autism candidate regions through analysis of reported cytogenetic abnormalities associated with autism. Mol. Psychiatry 1, 18-28. doi: 10.1038/sj.mp.4001757

Vuillermot, S., Luan, W., Meyer, U., and Eyles, D. (2017). Vitamin D treatment during pregnancy prevents autism-related phenotypes in a mouse model of maternal immune activation. Mol. Autism 8:9. doi: 10.1186/s13229-017-0125-0

Walum, H., Lichtenstein, P., Neiderhiser, J. M., Reiss, D., Ganiban, J. M., Spotts, E. L., et al. (2012). Variation in the oxytocin receptor gene is associated with pair-bonding and social behavior. Biol. Psychiatry 71, 419-426. doi: 10.1016/j.biopsych.2011.09.002

Wang, J., Qin, W., Liu, B., Wang, D., Zhang, Y., Jiang, T., et al. (2013). Variant in OXTR gene and functional connectivity of the hypothalamus in normal subjects. Neuroimage 81, 199-204. doi: 10.1016/j.neuroimage.2013.05.029

Wang, J., Qin, W., Liu, F., Liu, B., Zhou, Y., Jiang, T., et al. (2016). Sex-specific mediation effect of the right fusiform face area volume on the association between variants in repeat length of AVPR1A RS3 and altruistic behavior in healthy adults. Hum. Brain Mapp. 37, 2700-2709. doi: 10.1002/hbm.23203

Wang, K., Zhang, H., Ma, D., Bucan, M., Glessner, J. T., Abrahams, B. S., et al. (2009). Common genetic variants on 5 p14.1 associate with autism spectrum disorders. Nature 459, 528-533. doi: 10.1038/nature07999

Wassink, T. H., Piven, J., Vieland, V. J., Pietila, J., Goedken, R. J., Folstein, S. E., et al. (2004). Examination of AVPR1a as an autism susceptibility gene. Mol. Psychiatry 9, 968-972. doi: 10.1038/sj.mp.4001503

Watanabe, T., Otowa, T., Abe, O., Kuwabara, H., Aoki, Y., Natsubori, T., et al. (2017). Oxytocin receptor gene variations predict neural and behavioral response to oxytocin in autism. Soc. Cogn. Affect. Neurosci. 12, 496-506. doi: $10.1093 /$ scan/nsw150

Watson, K. K., and Platt, M. L. (2012). Of mice and monkeys: using nonhuman primate models to bridge mouse- and human-based investigations of autism spectrum disorders. J. Neurodev. Disord. 4:21. doi: 10.1186/1866-195 5-4-21

Weisman, O., Pelphrey, K. A., Leckman, J. F., Feldman, R., Lu, Y., Chong, A., et al. (2015). The association between $2 \mathrm{D}: 4 \mathrm{D}$ ratio and cognitive empathy is contingent on a common polymorphism in the oxytocin receptor gene (OXTR rs53576). Psychoneuroendocrinology 58, 23-32. doi: 10.1016/j.psyneuen.2015.04.007

Wermter, A. K., Kamp-Becker, I., Hesse, P., Schulte-Körne, G., Strauch, K., and Remschmidt, H. (2010). Evidence for the involvement of genetic variation in the oxytocin receptor gene (OXTR) in the etiology of autistic disorders on high-functioning level. Am. J. Med. Genet. Part B Neuropsychiatr. Genet. 153B, 629-639. doi: 10.1002/ajmg.b.31032

Wersinger, S. R., Kelliher, K. R., Zufall, F., Lolait, S. J., O'Carroll, A. M., and Young, S. W. (2004). Social motivation is reduced in vasopressin $1 \mathrm{~b}$ receptor null mice despite normal performance in an olfactory discrimination task. Horm. Behav. 46, 638-645. doi: 10.1016/j.yhbeh.2004.07.004

Winslow, J. T., and Insel, T. R. (2004). Neuroendocrine basis of social recognition. Curr. Opin. Neurobiol. 14, 248-253. doi: 10.1016/j.conb.2004.03.009

Winslow, J. T., and Insel, T. R. (2002). The social deficits of the oxytocin knockout mouse. Neuropeptides 36, 221-229. doi: 10.1054/npep.2002.0909

$\mathrm{Wu}, \mathrm{N} ., \mathrm{Li}, \mathrm{Z}$., and Su, Y. (2012). The association between oxytocin receptor gene polymorphism (OXTR) and trait empathy. J. Affect. Disord. 138, 468-472. doi: 10.1016/j.jad.2012.01.009

$\mathrm{Wu}, \mathrm{N}$., Shang, S., and Su, Y. (2015). The arginine vasopressin V1b receptor gene and prosociality: mediation role of emotional empathy. Psych J. 4, 160-165. doi: 10.1002/pchj.102

Wu, S., Jia, M., Ruan, Y., Liu, J., Guo, Y., Shuang, M., et al. (2005). Positive association of the oxytocin receptor gene (OXTR) with autism in the Chinese Han population. Biol. Psychiatry 58, 74-77. doi: 10.1016/j.biopsych.2005.03.013

Yamasue, H. (2013). Function and structure in social brain regions can link oxytocin-receptor genes with autistic social behavior. Brain Dev. 35, 111-118. doi: 10.1016/j.braindev.2012.08.010

Yang, S. Y., Cho, S. C., Yoo, H. J., Cho, I. H., Park, M., Kim, B. N., et al. (2010a). Association study between single nucleotide polymorphisms in promoter region of AVPR1A and Korean autism spectrum disorders. Neurosci. Lett. 479, 197-200. doi: 10.1016/j.neulet.2010.05.050

Yang, S. Y., Cho, S. C., Yoo, H. J., Cho, I. H., Park, M., Yoe, J., et al. (2010b). Familybased association study of microsatellites in the $5^{\prime}$ flanking region of AVPR1A with autism spectrum disorder in the Korean population. Psychiatry Res. 178, 199-201. doi: 10.1016/j.psychres.2009.11.007

Yang, S. Y., Kim, S. A., Hur, G. M., Park, M., Park, J. E., and Yoo, H. J. (2017) Replicative genetic association study between functional polymorphisms in AVPR1A and social behavior scales of autism spectrum disorder in the Korean population. Mol. Autism 8:44. doi: 10.1186/s13229-017-0161-9

Yee, N., Schwarting, R. K., Fuchs, E., and Wöhr, M. (2012). Increased affective ultrasonic communication during fear learning in adult male rats exposed to maternal immune activation. J. Psychiatr. Res. 46, 1199-1205. doi: 10.1016/j.jpsychires.2012.05.010

Yin, C. L., Chen, H. I., Li, L. H., Chien, Y. L., Liao, H. M., Chou, M. C., et al. (2016). Genome-wide analysis of copy number variations identifies PARK2 as a candidate gene for autism spectrum disorder. Mol. Autism 7:23. doi: 10.1186/s13229-016-0087-7

Yirmiya, N., Rosenberg, C., Levi, S., Salomon, S., Shulman, C., Nemanov, L., et al. (2006). Association between the arginine vasopressin la receptor (AVPR1a) gene and autism in a family-based study: mediation by socialization skills. Mol. Psychiatry 11, 488-494. doi: 10.1038/sj.mp.4001812

Ylisaukko-oja, T., Alarcón, M., Cantor, R. M., Auranen, M., Vanhala, R., Kempas, E., et al. (2006). Search for autism loci by combined analysis of Autism genetic resource exchange and finnish families. Ann. Neurol. 59, 145-155. doi: 10.1002/ana.20722

Young, L. J. (1999). Oxytocin and vasopressin receptors and species-typical social behaviors. Horm. Behav. 36, 212-221. doi: 10.1006/hbeh.1999.1548

Yrigollen, C. M., Han, S. S., Kochetkova, A., Babitz, T., Chang, J. T., Volkmar, F. R., et al. (2008). Genes controlling affiliative behavior as candidate genes for autism. Biol. Psychiatry 63, 911-916. doi: 10.1016/j.biopsych.2007.11.015

Zai, C. C., Muir, K. E., Nowrouzi, B., Shaikh, S. A., Choi, E., Berall, L., et al. (2012). Possible genetic association between vasopressin receptor $1 \mathrm{~B}$ and 
child aggression. Psychiatry Res. 200, 784-788. doi: 10.1016/j.psychres.2012. 07.031

Zhang, R., Zhang, H. F., Han, J. S., and Han, S. P. (2017). Genes related to oxytocin and arginine-vasopressin pathways: associations with autism spectrum disorders. Neurosci. Bull. 33, 238-246. doi: 10.1007/s12264-017-0 $120-7$

Zerbo, O., Iosif, A. M., Walker, C., Ozonoff, S., Hansen, R. L., and HertzPicciotto, I. (2013). Is maternal influenza or fever during pregnancy associated with autism or developmental delays? Results from the CHARGE (CHildhood Autism Risks from Genetics and Environment) study. J. Autism Dev. Dis. 43, 25-33. doi: 10.1007/s10803-012-1540-x

Zerbo, O., Qian, Y., Yoshida, C., Grether, J. K., Van de Water, J., and Croen, L. A. (2015). Maternal infection during pregnancy and autism spectrum disorders. J. Autism Dev. Disord. 45, 4015-4025. doi: 10.1007/s10803-0132016-3
Zimmermann, F. F., Gaspary, K. V., Siebel, A. M., and Bonan, C. D. (2016). Oxytocin reversed MK-801-induced social interaction and aggression deficits in zebrafish. Behav. Brain Res. 311, 368-374. doi: 10.1016/j.bbr.2016.05.059

Conflict of Interest Statement: The authors declare that the research was conducted in the absence of any commercial or financial relationships that could be construed as a potential conflict of interest.

Copyright (c) 2018 Cataldo, Azhari and Esposito. This is an open-access article distributed under the terms of the Creative Commons Attribution License (CC $B Y)$. The use, distribution or reproduction in other forums is permitted, provided the original author(s) and the copyright owner are credited and that the original publication in this journal is cited, in accordance with accepted academic practice. No use, distribution or reproduction is permitted which does not comply with these terms. 